\title{
LONG-LIVED COLLATERALIZED ASSETS AND BUBBLES
}

Autor: Aloisio Araujo, Mário Páscoa , Juan Pablo Torres-Martínez.

Santiago, Agosto 2008

La serie de Documentos de Trabajo (SDT) del Departamento de Economía de la Universidad de Chile en versión PDF puede descargarse en la dirección electrónica www.econ.uchile.cl/SDT . Para contactar al editor ejecutivo de SDT remitirse a sdt@econ.uchile.cl 


\title{
Serie Documentos de Trabajo \\ N 284
}

\section{Long-lived Collateralized Assets and Bubbles}

\author{
Aloisio Araujo Mário Páscoa Juan Pablo Torres-Martínez
}

\begin{abstract}
When infinite-lived agents trade long-lived assets secured by durable goods, equilibrium exists without any additional debt constraints or uniform impatience conditions on agents' characteristics. Also, regardless of whether assets' net supply is positive or zero, price bubbles are absent when physical endowments are uniformly bounded away from zero. Otherwise, bubbles may occur, even for assets in persistently positive net supply and for deflators yielding finite present values of aggregate wealth.
\end{abstract}

\section{Keywords:}

Collateralized assets, Existence of equilibrium, Asset pricing bubbles. 


\title{
LONG-LIVED COLLATERALIZED ASSETS AND BUBBLES
}

\author{
ALOISIO ARAUJO, MÁRIO R. PÁSCOA, AND JUAN PABLO TORRES-MARTÍNEZ
}

\begin{abstract}
When infinite-lived agents trade long-lived assets secured by durable goods, equilibrium exists without any additional debt constraints or uniform impatience conditions on agents' characteristics. Also, regardless of whether assets' net supply is positive or zero, price bubbles are absent when physical endowments are uniformly bounded away from zero. Otherwise, bubbles may occur, even for assets in persistently positive net supply and for deflators yielding finite present values of aggregate wealth.
\end{abstract}

Keywords. Collateralized assets, Existence of equilibrium, Asset pricing bubbles. JEL Classification. D50, D52.

\section{INTRODUCTION}

Sequential economies with infinite-lived assets have been studied for quite a long time in finance and in macroeconomics. The pioneering models were of two kinds: the overlapping generations models by Samuelson (1958) and Gale (1973) and the infinite-lived agents model by Bewley (1980). The latter inspired a general equilibrium literature that focused on two subtle issues: existence of equilibrium and occurrence of asset price bubbles (see, for instance, Magill and Quinzii (1996), Hernandez and Santos (1996), and Santos and Woodford (1997)).

The previous literature addressed the case of default-free unsecured assets. Generic existence of equilibrium was established under debt-constraints (Magill and Quinzii (1996) and Hernandez and Santos (1996)). For nicely behaved deflators yielding finite present values of wealth, speculation in assets in positive net supply was ruled out when markets were complete or when agents were uniformly impatient, but bubbles with real effects might occur in the case of assets in zero net supply (see Santos and Woodford (1997) and Magill and Quinzii (1996)).

When default is allowed but short-sales are secured by durable goods, the optimization problem of infinite lived agents gains a very nice structure that allows us to approach existence of equilibrium and speculation in a new way. In fact, the returns from past actions (namely from the joint operation of collateralizing and short-selling) are always non-negative and, therefore, as in positive dynamic programming, Euler and transversality conditions are not just necessary but also sufficient for individual optimality. Moreover, endowments are no longer required to be bounded away from zero, due to the durability of previous endowments.

Date: July, 2008.

We benefited from comments on earlier versions presented at the SAET 2003 conference in Rhodes, the SAET conference at Purdue University (2005) and NBER Debreu memorial conference at U.C. Berkeley (2005). A.Araujo and J.P.Torres-Martínez acknowledge financial support from CNPq and Faperj. M.R.Páscoa benefited from a Nova Forum grant and support from FCT and FEDER through project POCTI/ECO 44386/2002. 
From the sufficiency of the optimality conditions we establish existence of equilibrium, without imposing debt constraints or uniform impatience requirements. It also gives us an easy way to construct examples of equilibrium: we just need to check Euler, transversality and market clearing conditions. That is, as in the case of short-lived assets (see Araujo, Páscoa and Torres-Martínez (2002) or Kubler and Schmedders (2003)), collateral avoids Ponzi schemes. Note that, these schemes were not the only possible reason for non-existence of equilibrium with long-lived assets. In fact, in economies with default-free long-lived assets, where those debt requirements were imposed, equilibrium still failed to exist and only generic existence was guaranteed (see Hernandez and Santos (1996) or Magill and Quinzii (1996)). Two difficulties came up: (i) there were no endogenous upper bounds on short-sales, as the rank of returns matrices became dependent on asset prices; and (ii) finite asset prices might be incompatible with non-arbitrage conditions, as the return matrices of zero-net supply assets could be unbounded along the event-tree (see Hernandez and Santos (1996, Example 3.9)). Collateral avoids also these two additional difficulties, since the scarcity of physical goods assures that collateralized short-sales are bounded (overcoming (i)) and, by non-arbitrage (see below), bounded collateral coefficients end up bounding asset prices (overcoming (ii)).

From the necessity of the optimality conditions we establish the properties that commodity and asset prices should satisfy and find out that asset prices are always bounded by the collateral cost. We use this result and focus on deflators that are compatible with the optimality conditions (and, therefore, known to yield finite present values of wealth). First, we show that mortgages, whose collateral does not have margin calls, are free of price bubbles unless the durable good serving as collateral (or being part of the real payments) has a price bubble itself. Secondly, for more general collateral requirements, speculation is ruled out if endowments are uniformly bounded away from zero, irrespective of whether the net supply of the asset is positive or zero. However, having dispensed with uniform impatience, for equilibrium purposes, endowments do not need to be uniformly bounded away from zero and, therefore, it becomes possible to construct examples of incomplete markets equilibrium with a positive price for an asset yielding no dividends, even when present values of wealth are finite. In these examples, collateral constraints are not binding and, therefore, the positive price is not due to a positive fundamental value consisting of shadow prices, but is rather due to a bubble. It is the diversity of individual deflators that allows for the positive net supply of the asset changes hands in such a way that the asset has a limiting positive price even if no one holds limiting long positions (as required by the transversality conditions).

Also, when individuals' inter-temporal marginal rates of substitution coincide, we prove that assets in persistently positive net supply are free of price bubbles. However, as a consequence of collateral seizure, the asset net supply is endogenous in our model and we may have bubbles if the net supply is asymptotically zero as a result of collateral seizure.

Finally, note that uniform impatience had played a crucial role in default-free economies when it came to show that debt constraints turned out to be equivalent to imposing the transversality requirements that the optimal plan should verify. That is, under uniform impatience, the chosen default-free plan was optimal among the debt-constrained or transversality-constrained plans that satisfied the budget constraints. In our model, the chosen plan is optimal among all bugdet feasible 
plans and we can do without uniform impatience, which is far from being a trivial assumption. Even for separable utility functions and endowments that are uniformly bounded away from zero, the assumption may fail if inter-temporal discounting is not stationary.

The rest of the paper is organized as follows. The next two sections present the model. In Section 4 we discuss a crucial property of the default model: a consumption and portfolio plan is individually optimal if and only if it satisfies Euler inequalities and a transversality condition on its cost. The necessity part is used to characterize asset prices. The sufficiency part, which is the novel result, is used to establish existence of equilibrium without uniform impatience requirements (in Section 5). Our asset pricing characterization (which is analogous to the non-arbitrage valuation studied by Araujo, Fajardo and Páscoa (2005)) is the basis for the definitions of fundamental values and for the results on absence of price bubbles (in Section 6). We close the paper with examples (Section 7) of asset price bubbles.

\section{Infinite Horizon Collateralized Asset Markets}

Uncertainty. We consider a discrete time economy with infinite horizon. A date is an element $t \in\{0,1, \ldots\}$. There is no uncertainty at $t=0$ and given a history of realization of the states of nature for the first $t$ dates, with $t \geq 1, \bar{s}_{t}=\left(s_{0}, \ldots, s_{t-1}\right)$, there is a finite set $S\left(\bar{s}_{t}\right)$ of states of nature that may occur at date $t$.

A vector $\xi=\left(t, \bar{s}_{t}, s\right)$, where $t \geq 1$ and $s \in S\left(\bar{s}_{t}\right)$, is called a node of the economy. There is only one node at $t=0$, that is denoted by $\xi_{0}$. Given $\xi=\left(t, \bar{s}_{t}, s\right)$ and $\mu=\left(t^{\prime}, \bar{s}_{t^{\prime}}, s^{\prime}\right)$, we say that $\mu$ is a successor of $\xi$, and write $\mu \geq \xi$, if both $t^{\prime} \geq t$ and $\left(\bar{s}_{t^{\prime}}, s^{\prime}\right)=\left(\bar{s}_{t}, s, \ldots\right)$. We write $\mu>\xi$ to say that $\mu \geq \xi$ but $\mu \neq \xi$. The set of nodes, called the event-tree, is denoted by $D$.

Let $t(\xi)$ be the date associated with a node $\xi \in D$. Let $\xi^{+}:=\{\mu \in D:(\mu \geq \xi) \wedge(t(\mu)=t(\xi)+1)\}$. The (unique) predecessor of $\xi$, with $t(\xi) \geq 1$, is denoted by $\xi^{-}$and $D(\xi)=\{\mu \in D: \mu \geq \xi\}$ is the subtree with root $\xi$. The family of nodes with date $T$ in $D(\xi)$ is denoted by $D_{T}(\xi)$. Finally, given $T \geq 1$, let $D^{T}(\xi):=\bigcup_{k=t(\xi)}^{T} D_{k}(\xi), D^{T}:=D^{T}\left(\xi_{0}\right)$ and $D_{T}:=D_{T}\left(\xi_{0}\right)$.

Physical markets. At each node there is a finite ordered set of commodities, $L$, which can be traded and may suffer transformations at the immediate successors nodes. We allow for goods that are perishable or perfectly durable and also for transformation of some commodities into others.

More formally, for any $\eta \in D$, there is a matrix with non-negative entries $Y_{\eta}=\left(Y_{\eta}\left(l, l^{\prime}\right)\right)_{\left(l, l^{\prime}\right) \in L \times L}$ such that, if one unit of good $l \in L$ is consumed at a node $\xi$, then at each $\mu \in \xi^{+}$remain $Y_{\mu}(l, l)$ units of $l$ and we obtain $Y_{\mu}\left(l^{\prime}, l\right)$ units of each commodity $l^{\prime} \neq l$. For convenience of notations, given a history of nodes $\left\{\xi_{1}, \ldots, \xi_{n}\right\}$, with $\xi_{j+1} \in \xi_{j}^{+}$, we define $Y_{\xi_{1}, \xi_{n}}$ as equal to $Y_{\xi_{n}} Y_{\xi_{n-1}} \cdots Y_{\xi_{2}}$, when $n>1$; and equal to the identity matrix when $n=1$.

Spot markets for commodity trade are available at each node. Denote by $p_{\xi}=\left(p_{\xi, l}: l \in L\right) \in \mathbb{R}_{+}^{L}$ the row vector of spot prices at $\xi \in D$ and by $p=\left(p_{\xi}: \xi \in D\right)$ the process of commodity prices. 
Financial markets. There is a finite ordered set $J$ of different types of infinite-lived securities. Assets may suffer default but are protected by physical collateral requirements. ${ }^{1}$ Assets of a given type have the same promises of real deliveries and the same collateral requirements. Thus, in the absence of default, assets of the same type can be treated as being the same security. However, when an asset issued at $\xi$ defaults at a successor node $\mu>\xi$, it converts into the respective garnishable collateral. For this reason, we suppose that, at every node, an asset of each type $j \in J$ can be issued. In this way, we assure that agents can constitute, at any node, new long or short positions on assets of any type. For the sake of simplicity, whenever there is no possible confusion, we will refer to an asset of type $j$ simply as asset $j$.

The net supply of $j \in J$ at $t=0$ is $e_{j} \geq 0$. At any $\xi>\xi_{0}$, real promises are given by a bundle $A(\xi, j) \in \mathbb{R}_{+}^{L}$. Let $\left(C_{\xi, j} ; \xi \in D\right) \in \mathbb{R}_{+}^{L \times D}$ be the plan of asset' $j$ unitary collateral requirements.

We denote by $q_{\xi}=\left(q_{\xi, j}, j \in J\right) \in \mathbb{R}_{+}^{J}$ the row vector of asset prices at $\xi \in D$, and by $q=\left(q_{\xi}, \xi \in\right.$ $D)$ a plan of asset prices in the event-tree.

Note that, holders of asset endowments are not required to constitute collateral when selling these endowments. However, when assets are short-sold, borrowers have to constitute collateral. Now, when purchasing an unit of an asset it is not possible to distinguish whether this unit was short-sold or is part of someone's endowment. Thus, the price and the return from this purchase will be the same in both cases. ${ }^{2}$

In case of default, the depreciated collateral will be seized. Also, others goods delivered by the collateral bundle may also be garnishable. That is, we assume that, in case of default on asset $j$ at node $\xi>\xi_{0}$, markets seize the garnishable collateral, which is given by a bundle $\widehat{C}_{\xi, j}$ that satisfies, $Y_{\xi}(l, l) C_{\xi^{-}, j, l} \leq \widehat{C}_{\xi, j, l} \leq Y_{\xi}(l, \cdot) C_{\xi^{-}, j}, \forall l \in L$. Note that, if $Y_{\xi}$ is a diagonal matrix (as in Araujo, Páscoa and Torres-Martínez (2002)), then $\widehat{C}_{\xi, j}$ coincides with $Y_{\xi} C_{\xi^{-}, j}$. However, when collateral is durable but delivers also perishable commodities at the next nodes, those deliveries might also be or not be seized in case of default. Hence, borrowers will pay and lenders expect to receive the minimum between the value of the garnishable collateral and the market value of the original debt. Thus, the (unitary) nominal payment made by asset $j$ at node $\xi>\xi_{0}$ is given by $D_{\xi, j}(p, q):=\min \left\{p_{\xi} A(\xi, j)+q_{\xi, j}, p_{\xi} \widehat{C}_{\xi, j}\right\}$. To shorten notations, let $D_{\xi}(p, q):=\left(D_{\xi, j}(p, q), j \in J\right)$.

Finally, we want to show two simple and important examples of collateral requirements processes contemplated by our framework. First, if for any $\xi \in D, C_{\xi, j}=C \in \mathbb{R}_{+}^{L}$, then, as collateral guarantees may depreciate along the event-tree, borrowers may need to buy additional physical resources in order to maintain their original short-positions. In some sense, it is similar to the well known market practice of margin calls. Secondly, the case of mortgage loans, where $C_{\xi, j} \leq Y_{\xi} C_{\xi^{-}, j}$, for any $\xi>\xi_{0}$. In this case, short-positions can be maintained without need to update the amount of physical guarantees.

\footnotetext{
${ }^{1}$ We could have allowed for price dependent collateral requirements and for financial collateral as long as we ruled out self-collateralization (the possibility that an asset ends of securing itself though a chain of other assets). For more details see Araujo, Páscoa and Torres-Martínez (2005)

${ }^{2}$ In other words, a holder of an asset endowment holds units of the tradeable asset subject to default and not of the underlying primitive asset free of default with promises $\left(A(\xi, j) ; \xi>\xi_{0}\right)$, which is not tradable.
} 
Households. There is a finite set, $H$, of infinite-lived agents that consume commodities and trade assets along the event-tree. Each agent $h \in H$ has financial endowments $e^{h}=\left(e_{j}^{h}\right)_{j \in J} \in \mathbb{R}_{+}^{J}$ at $t=0$, which satisfy $e^{j}=\sum_{h \in H} e_{j}^{h}$. Physical endowment processes are given by $w^{h}=\left(w_{\xi}^{h} ; \xi \in D\right) \in$ $\mathbb{R}_{+}^{D \times L}$. At each $\xi \in D$, any agent $h$ can choose a plan $z_{\xi}^{h}=\left(x_{\xi}^{h}, \theta_{\xi}^{h}, \varphi_{\xi}^{h}\right) \geq 0$, where $x_{\xi}^{h}:=\left(x_{\xi, l}^{h} ; l \in L\right)$ is the autonomous consumption bundle (that is, her consumption in excess of required physical collateral) and $\left(\theta_{\xi}^{h}, \varphi_{\xi}^{h}\right)=\left(\left(\theta_{\xi, j}^{h}, \varphi_{\xi, j}^{h}\right) ; j \in J\right)$ denotes, respectively, her long-and short-positions at $\xi$. Agent $h$ consumption at a node $\xi$ is given by $\widehat{x}_{\xi}^{h}=x_{\xi}^{h}+\sum_{j \in J} C_{\xi, j} \varphi_{\xi, j}^{h}$.

Given prices $(p, q)$, the objective of consumer $h$ is to maximize her utility function $U^{h}: \mathbb{R}_{+}^{D \times L} \rightarrow$ $\mathbb{R}_{+} \cup\{+\infty\}$ over the plans $\widehat{x}^{h}$, by choosing a plan $z^{h}=\left(x^{h}, \theta^{h}, \varphi^{h}\right) \in \mathbb{E}:=\mathbb{R}_{+}^{D \times L} \times \mathbb{R}_{+}^{D \times J} \times \mathbb{R}_{+}^{D \times J}$ which satisfies the following budget constraints, ${ }^{3}$

$$
g_{\xi_{0}}^{h}\left(z_{\xi_{0}}^{h}, z_{\xi_{0}^{-}}^{h} ; p, q\right):=p_{\xi_{0}}\left(\widehat{x}_{\xi_{0}}^{h}-w_{\xi_{0}}^{h}\right)+q_{\xi_{0}}\left(\theta_{\xi_{0}}^{h}-\varphi_{\xi_{0}}^{h}-e^{h}\right) \leq 0,
$$

and for all $\xi>\xi_{0}$,

$$
g_{\xi}^{h}\left(z_{\xi}^{h}, z_{\xi^{-}}^{h} ; p, q\right):=p_{\xi}\left(\widehat{x}_{\xi}^{h}-w_{\xi}^{h}-Y_{\xi} \widehat{x}_{\xi^{-}}^{h}\right)+q_{\xi}\left(\theta_{\xi}^{h}-\varphi_{\xi}^{h}\right)-D_{\xi}(p, q)\left(\theta_{\xi^{-}}^{h}-\varphi_{\xi^{-}}^{h}\right) \leq 0,
$$

where $z_{\xi_{0}^{-}}^{h}:=\left(x_{\xi_{0}^{-}}^{h}, \theta_{\xi_{0}^{-}}^{h}, \varphi_{\xi_{0}^{-}}^{h}\right)=0$. The budget set of agent $h$ at prices $(p, q)$, denoted by $B^{h}(p, q)$, is the collection of plans $(x, \theta, \varphi) \in \mathbb{E}$ such that inequalities (1) and (2) hold. Moreover, without loss of generality, we restrict the price set to $\mathbb{P}:=\left\{\left(p_{\xi}, q_{\xi}\right)_{\xi \in D}:\left(p_{\xi}, q_{\xi}\right) \in \Delta_{+}^{L+J-1}, \forall \xi \in D\right\}$, where $\Delta_{+}^{n-1}$ denotes the $(n-1)$-dimensional simplex in $\mathbb{R}_{+}^{n}$.

Market clearing. Real returns from asset endowments have to be taken into account in the market clearing conditions. When an asset does not default, the real returns from asset endowments coincide with the promised real returns. In this first case, the asset will remain with the same positive net supply that it had at the preceding node. However, in the case of default, real returns generated by assets' endowments will be determined by garnishable collateral coefficients. In this second case, the asset can be traded again, as long as the collateral requirements are again satisfied, but the positive net financial supply disappears. In fact, the previous positive net supply has been entirely converted into a supply of garnished collateral.

In the borderline case, when borrowers are indifferent between surrendering the garnishable collateral and honoring the promise, in value terms it does not matter whether the collateral is garnished or the promise is payed, but, for the purposes of market clearing, this choice becomes relevant. This choice will determine also whether the asset's net supply will decrease or not.

Given $(p, q) \in \mathbb{P}$, we introduce, at each $\xi \neq \xi_{0}$, delivery rates $\lambda_{\xi, j} \in[0,1]$, which are equal to one when the promise is lower than the value of the garnishable collateral, equal to zero when the opposite strict inequality holds, but may take a value between zero and one in case of equality. ${ }^{4}$

\footnotetext{
${ }^{3}$ Note that, the non-negativity condition on the autonomous consumption represents the physical collateral constraint. In fact, the later requires $\widehat{x}_{\xi}^{h} \geq \sum_{j \in J} C_{\xi, j} \varphi_{\xi, j}^{h}$, which is equivalent to $x_{\xi}^{h} \geq 0$.

${ }^{4}$ Since the promise and the garnishable collateral coefficients are impersonal, the delivery rates may vary across agents but there is no rationale for such differences. Hence, we can concentrate our attention on outcomes where, in the case of indifference between paying the promise and surrendering the garnishable collateral, all agents choose the same combination of these two, that is, the same delivery rates.
} 
Using the delivery rates $\lambda_{\xi}=\left(\lambda_{\xi, j}\right)_{j \in J}$, the effective nominal return of an asset $j$ in positive net supply, $D_{\xi, j}(p, q)$, can be seen as the value of a real component plus the value of a financial position, $D_{\xi, j}(p, q)=p_{\xi}\left(\lambda_{\xi, j} A(\xi, j)+\left(1-\lambda_{\xi, j}\right) \widehat{C}_{\xi, j}\right)+q_{\xi} \lambda_{\xi, j}$, where the real component is either the promised physical delivery or the garnishable collateral or a combination of the two.

Definition 1. An equilibrium for our economy is given by prices $(p, q) \in \mathbb{P}$, individual plans $\left(\left(x^{h}, \theta^{h}, \varphi^{h}\right)\right)_{h \in H} \in \mathbb{E}^{H}$ and delivery rates $\lambda \in[0,1]^{\left(D \backslash\left\{\xi_{0}\right\}\right) \times J}$, such that

A. For each $h \in H,\left(x^{h}, \theta^{h}, \varphi^{h}\right) \in \operatorname{Argmax}\left\{U^{h}(\widehat{x}),(x, \theta, \varphi) \in B^{h}(p, q)\right\}$.

B. At each node $\xi \neq \xi_{0}, D_{\xi, j}(p, q)=\lambda_{\xi, j}\left(p_{\xi} A(\xi, j)+q_{\xi, j}\right)+\left(1-\lambda_{\xi, j}\right) p_{\xi} \widehat{C}_{\xi, j}, \forall j \in J$.

C. Asset markets are cleared. That is, for each $j \in J$,

$$
\begin{aligned}
\sum_{h \in H}\left(\theta_{\xi_{0}, j}^{h}-\varphi_{\xi_{0}, j}^{h}\right) & =e_{j} \\
\sum_{h \in H}\left(\theta_{\xi, j}^{h}-\varphi_{\xi, j}^{h}\right) & =\lambda_{\xi, j} \sum_{h \in H}\left(\theta_{\xi^{-}, j}^{h}-\varphi_{\xi^{-}, j}^{h}\right), \quad \forall \xi \neq \xi_{0} .
\end{aligned}
$$

D. Physical markets are cleared.

$$
\sum_{h \in H} \widehat{x}_{\xi_{0}}^{h}=\sum_{h \in H} w_{\xi_{0}}^{h}
$$

and, at each $\xi \neq \xi_{0}$,

$$
\sum_{h \in H} \widehat{x}_{\xi}^{h}=\sum_{h \in H}\left(w_{\xi}^{h}+Y_{\xi} \widehat{x}_{\xi^{-}}^{h}\right)+\sum_{j \in J}\left(\lambda_{\xi, j} A(\xi, j)+\left(1-\lambda_{\xi, j}\right) \widehat{C}_{\xi, j}\right) \sum_{h \in H}\left(\theta_{\xi^{-}, j}^{h}-\varphi_{\xi^{-}, j}^{h}\right) .
$$

\section{Assumptions on Agents' Characteristics}

As commodities can be durable goods, the traditional assumption that individual endowments of commodities are interior points can be replaced by the weaker assumption that requires only individual accumulated resources to be interior points. Moreover, to assure the existence of equilibrium, we do not need to impose any uniform lower bound in the aggregate cumulated resources. Thus we allow for durable commodities whose aggregate resources converge to zero.

Assumption A. For each $(h, \xi) \in H \times D$, given the history of realization of states of nature up to node $\xi, F_{\xi}:=\left\{\xi_{0}, \ldots, \xi^{-}, \xi\right\}$, we have that $W_{\xi}^{h}:=\sum_{\mu \in F_{\xi}} Y_{\mu, \xi} w_{\mu}^{h} \gg 0$. Moreover, for each $(\xi, j) \in D \times J, C_{\xi, j} \neq 0$.

The aggregated resources up to a node $\xi$ need to take into account the streams of real resources generated by the financial endowments. Thus, an upper bound for the bundle of aggregate physical resources up to a node $\xi$ is given by $\mathbb{W}_{\xi}:=\sum_{h \in H} W_{\xi}^{h}+\sum_{\mu \in F_{\xi}} Y_{\mu, \xi} \sum_{j \in J} b_{\mu}^{j} e_{j}$, where $b_{\xi_{0}}^{j}=0$ and $b_{\xi}^{j}=\left(b_{\xi, l}^{j}\right)_{l \in L}$, with $b_{\xi, l}^{j}=\max \left\{\widehat{C}_{\xi, j, l} ; A(\xi, j)_{l}\right\}$, for each $\xi>\xi_{0}$.

Assumption B. The utility function of each $h \in H$ is separable in time and in states of nature, in the sense that $U^{h}(\widehat{x}):=\sum_{\xi \in D} u_{\xi}^{h}(\widehat{x}(\xi))$, where functions $u_{\xi}^{h}: \mathbb{R}_{+}^{L} \rightarrow \mathbb{R}_{+}$are strictly concave, continuous, and strictly increasing. Also, $\sum_{\xi \in D} u_{\xi}^{h}\left(\mathbb{W}_{\xi}\right)<+\infty$. 
Under hypotheses above, uniform impatience conditions imposed by Hernandez and Santos (1996, Assumption C.3), Magill and Quinzzi (1996, Assumptions B2 and B4) and Santos and Woodford (1997, Assumption A.2) do not necessarily hold. ${ }^{5}$ For example, given any $u: \mathbb{R}_{+}^{L} \rightarrow \mathbb{R}_{+}$strictly concave, continuous, and strictly increasing, consider the function $U(\widehat{x}):=\sum_{\xi \in D} \beta_{t(\xi)} \rho(\xi) u(\widehat{x}(\xi))$, where $\left(\beta_{t}\right)_{t \geq 0} \in \mathbb{R}_{++}^{\mathbb{N}}, \rho\left(\xi_{0}\right)=1$ and, for each $\xi \in D, \rho(\xi)=\sum_{\mu \in \xi^{+}} \rho(\mu)$. Then, when physical resources are uniformly bounded along the event-tree and $\sum_{t \geq 0} \beta_{t}$ is finite, Assumption B holds. If in addition individual endowments are uniformly bounded away from zero, Assumption A is satisfied. However, in this context, the function $U$ may fail to satisfy uniform impatience condition when inter-temporal discount factors are not stationary. Santos and Woodford (1997, example 4.5) gave an example that illustrates this possibility.

\section{Individual Optimality}

In this section we present necessary and sufficient conditions for individual optimality. As in positive dynamic programming theory, we will show that the default structure gives inter-temporal Lagrangian functions a sign property under which Euler inequalities jointly with a transversality condition are not just necessary but also sufficient to guarantee the optimality of a consumptionportfolio plan.

Let $\mathbb{Z}:=\mathbb{R}^{L} \times \mathbb{R}^{J} \times \mathbb{R}^{J}$. Given prices $(p, q) \in \mathbb{P}$, it follows from the arguments of the previous section that the objective of the agent $h$ is to find a plan $\left(z_{\xi}^{h}\right)_{\xi \in D} \in \mathbb{Z}^{D}$ in order to solve

$$
\begin{aligned}
P_{(p, q)}^{h} \max & \sum_{\xi \in D} v_{\xi}^{h}\left(z_{\xi}\right) \\
\text { s.t. } & \left\{\begin{array}{l}
g_{\xi}^{h}\left(z_{\xi}, z_{\xi} ; p, q\right) \leq 0, \quad \forall \xi \in D, \\
z_{\xi}=\left(x_{\xi}, \theta_{\xi}, \varphi_{\xi}\right) \geq 0, \quad \forall \xi \in D, \quad z_{\xi_{0}^{-}}=0 .
\end{array}\right.
\end{aligned}
$$

where $v_{\xi}^{h}: \mathbb{Z} \rightarrow \mathbb{R} \cup\{-\infty\}$ is defined at any $z_{\xi}=\left(x_{\xi}, \theta_{\xi}, \varphi_{\xi}\right) \in \mathbb{Z}$ by

$$
v_{\xi}^{h}\left(z_{\xi}\right)= \begin{cases}u_{\xi}^{h}\left(x_{\xi}+\sum_{j \in J} C_{\xi, j} \varphi_{\xi, j}\right) & \text { if } x_{\xi}+\sum_{j \in J} C_{\xi, j} \varphi_{\xi, j} \geq 0 \\ -\infty & \text { in other case. }\end{cases}
$$

For each real number $\gamma \geq 0$, let $\mathcal{L}_{\xi}^{h}(\cdot, \gamma ; p, q): \mathbb{Z} \times \mathbb{Z} \rightarrow \mathbb{R}$ be the Lagrangian function associated to consumer problem at node $\xi$, which is defined by

$$
\mathcal{L}_{\xi}^{h}\left(z_{\xi}, z_{\xi^{-}}, \gamma ; p, q\right)=v_{\xi}^{h}\left(z_{\xi}\right)-\gamma g_{\xi}^{h}\left(z_{\xi}, z_{\xi^{-}} ; p, q\right)
$$

Since under Assumption B the function $\mathcal{L}_{\xi}^{h}(\cdot, \gamma ; p, q)$ is concave, we can consider its super-differential set at any point $\left(z_{\xi}, z_{\xi^{-}}\right) \in \mathbb{Z} \times \mathbb{Z}, \partial \mathcal{L}_{\xi}^{h}\left(z_{\xi}, z_{\xi^{-}}, \gamma ; p, q\right)$, which is defined as the set of vectors

\footnotetext{
${ }^{5}$ For instance, using the notation of Assumption B, in a context where aggregated physical endowments were exogenously fixed and given by the plan $\left(W_{\xi}\right)_{\xi \in D}$, Hernandez and Santos (1996) imposed the following assumption of uniform impatience: There exists $\sigma \times K \in[0,1) \times \mathbb{R}_{++}$such that, for any plan of consumption $\left(\widehat{x}_{\xi}\right) \xi \in D$ for which $\widehat{x}_{\xi} \leq W_{\xi}, \forall \xi \in D$, we have that

$$
u_{\xi}^{h}\left(\widehat{x}_{\xi}+K W_{\xi}\right)+\sum_{\mu>\xi} u_{\mu}^{h}\left(\sigma \widehat{x}_{\mu}\right)>\sum_{\mu \geq \xi} u_{\mu}^{h}\left(\widehat{x}_{\mu}\right), \quad \forall h \in H .
$$
}


$\left(\mathcal{L}_{\xi, 1}^{\prime}, \mathcal{L}_{\xi, 2}^{\prime}\right) \in \mathbb{Z} \times \mathbb{Z}$ such that, for all pair $\left(z_{\xi}^{\prime}, z_{\xi^{-}}^{\prime}\right) \in \mathbb{Z} \times \mathbb{Z}$

$$
\mathcal{L}_{\xi}^{h}\left(z_{\xi}^{\prime}, z_{\xi^{-}}^{\prime}, \gamma ; p, q\right)-\mathcal{L}_{\xi}^{h}\left(z_{\xi}, z_{\xi^{-}}, \gamma ; p, q\right) \leq\left(\mathcal{L}_{\xi, 1}^{\prime}, \mathcal{L}_{\xi, 2}^{\prime}\right) \cdot\left(\left(z_{\xi}^{\prime}, z_{\xi^{-}}^{\prime}\right)-\left(z_{\xi}, z_{\xi^{-}}\right)\right)
$$

Essentially, the above vectors $\mathcal{L}_{\xi, 1}^{\prime}$ and $\mathcal{L}_{\xi, 2}^{\prime}$ are partial super-gradients with respect to the current and past decision variables, respectively.

Definition 2. Given $(p, q) \in \mathbb{P},\left(\gamma_{\xi}^{h}\right)_{\xi \in D} \in \mathbb{R}_{++}^{D}$ is a plan of Kuhn-Tucker multipliers associated with $\left(z_{\xi}^{h}\right)_{\xi \in D} \in \mathbb{Z}^{D}$ if there is $\left(\mathcal{L}_{\xi, 1}^{\prime}, \mathcal{L}_{\xi, 2}^{\prime}\right)_{\xi \in D} \in \prod_{\xi \in D} \partial \mathcal{L}_{\xi}^{h}\left(z_{\xi}^{h}, z_{\xi^{-}}^{h}, \gamma_{\xi}^{h} ; p, q\right)$ such that, for any $\xi \in D$, $\gamma_{\xi}^{h} g_{\xi}^{h}\left(z_{\xi}^{h}, z_{\xi^{-}}^{h} ; p, q\right)=0$ and the following transversality and Euler conditions hold,

$$
\lim _{T \rightarrow+\infty} \sum_{\mu \in D_{T}\left(\xi_{0}\right)} \mathcal{L}_{\mu, 1}^{\prime} z_{\mu}^{h}=0 .
$$

$$
\mathcal{L}_{\xi, 1}^{\prime}+\sum_{\mu \in \xi^{+}} \mathcal{L}_{\mu, 2}^{\prime} \leq 0, \quad\left(\mathcal{L}_{\xi, 1}^{\prime}+\sum_{\mu \in \xi^{+}} \mathcal{L}_{\mu, 2}^{\prime}\right) z_{\xi}^{h}=0, \quad \forall \xi \in D
$$

Proposition 1. Suppose that Assumptions $A$ and $B$ hold. Given $(p, q) \in \mathbb{P}$, take a plan $\left(z_{\xi}^{h}\right)_{\xi \in D}=$ $\left(x_{\xi}^{h}, \theta_{\xi}^{h}, \varphi_{\xi}^{h}\right)_{\xi \in D} \in B^{h}(p, q)$.

(i) If $\left(z_{\xi}^{h}\right)_{\xi \in D}$ gives a finite optimum to $P_{(p, q)}^{h}$, then there is a plan of Kuhn-Tucker multipliers associated with $\left(z_{\xi}^{h}\right)_{\xi \in D \text {. }}$

(ii) Reciprocally, the plan $\left(z_{\xi}^{h}\right)_{\xi \in D}$ solves $P_{(p, q)}^{h}$ when there are Kuhn-Tucker multipliers associated with it. Also, if $\widehat{x}_{\xi}^{h} \leq \mathbb{W}_{\xi}$, for each $\xi \in D$, then the optimum value is finite.

(iii) Given Kuhn-Tucker multipliers, $\left(\gamma_{\xi}^{h}\right)_{\xi \in D}$, associated with $\left(z_{\xi}^{h}\right)_{\xi \in D}, \sum_{\xi \in D} \gamma_{\xi}^{h} p_{\xi} w_{\xi}^{h}<\infty$.

The proof that existence of Kuhn-Tucker multipliers implies individual optimality depends crucially on the following sign property of Lagrangian functions, which holds at any $\xi \in D$ : Given prices $(p, q) \in \mathbb{P}$ and a plan $\left(z_{\xi}\right)_{\xi \in D} \in \mathbb{Z}^{D}$,

$$
\forall \gamma \in \mathbb{R}_{++} \forall \xi>\xi_{0}: \quad\left(\mathcal{L}_{\xi, 1}^{\prime}, \mathcal{L}_{\xi, 2}^{\prime}\right) \in \partial \mathcal{L}_{\xi}^{h}\left(z_{\xi}, z_{\xi^{-}}, \gamma ; p, q\right) \Longrightarrow \mathcal{L}_{\xi, 2}^{\prime} \geq 0
$$

This property is very specific to our model. In fact, as for each $j \in J$ effective returns $D_{\xi, j}(p, q)$ are not greater than the respective garnishable collateral values, the joint returns from actions taken at immediately preceding nodes are non-negative (for more details, see Appendix A).

Condition (TC) is not a constraint that is imposed together with the budget restrictions (as was the case in Hernandez and Santos (1996) or Magill and Quinzii (1996)), it is rather a property that optimal plans should satisfy. Moreover, as the deflated value of endowments is summable (item (iii) of Proposition 1) condition (TC) can be rewritten as requiring that, as time tends to infinity, the deflated cost of the autonomous consumption goes to zero,

$$
\lim _{T \rightarrow+\infty} \sum_{\xi \in D_{T}\left(\xi_{0}\right)} \gamma_{\xi}^{h} p_{\xi} x_{\xi}^{h}=0
$$


jointly with the cost of the joint operation of constituting collateral and short-selling,

$$
\lim _{T \rightarrow+\infty} \sum_{\xi \in D_{T}\left(\xi_{0}\right)} \gamma_{\xi}^{h}\left(p_{\xi} \sum_{j \in J} C_{\xi, j} \varphi_{\xi, j}^{h}-q_{\xi} \varphi_{\xi}^{h}\right)=0 ;
$$

and the cost of asset purchases,

$$
\lim _{T \rightarrow+\infty} \sum_{\xi \in D_{T}\left(\xi_{0}\right)} \gamma_{\xi}^{h} q_{\xi} \theta_{\xi}^{h}=0
$$

where $z_{\xi}^{h}=\left(x_{\xi}^{h}, \theta_{\xi}^{h}, \varphi_{\xi}^{h}\right)$ (see Appendix A).

We end this section with a characterization of commodity and asset prices.

Corollary 1. (Asset pricing Conditions) Suppose that Assumptions $A$ and B hold. Fix prices $(p, q) \in \mathbb{P}$ such that, for some $h \in H, P_{(p, q)}^{h}$ has a finite optimum. Then, there exist, for any $\xi \in D$, strictly positive deflators $\gamma_{\xi}$ and non-pecuniary returns $\alpha_{\xi}=\left(\alpha_{\xi, l}\right)_{l \in L} \in \mathbb{R}_{++}^{L}$ such that, for each $(l, j) \in L \times J$,

$$
\begin{aligned}
\gamma_{\xi} p_{\xi, l} & \geq \sum_{\mu \in \xi^{+}} \gamma_{\mu} p_{\mu} Y_{\mu}(\cdot, l)+\alpha_{\xi, l} ; \\
\gamma_{\xi} q_{\xi, j} & \geq \sum_{\mu \in \xi^{+}} \gamma_{\mu} D_{\mu, j}(p, q) ; \\
\gamma_{\xi}\left(p_{\xi} C_{\xi, j}-q_{\xi, j}\right) & \geq \sum_{\mu \in \xi^{+}} \gamma_{\mu}\left(p_{\mu} Y_{\mu} C_{\xi, j}-D_{\mu, j}(p, q)\right)+\alpha_{\xi} C_{\xi, j} .
\end{aligned}
$$

Moreover, for any $(\xi, j) \in D \times J$, conditions (6) or (7) are strict inequalities only when inequality (5) is strict for some $l \in L$ for which $C_{\xi, j, l}>0$.

This result is a direct consequence of the existence of Kuhn-Tucker multipliers associated with agent $h$ optimal problem. Indeed, as we prove in Appendix A, conditions (5)-(7) are essentially equal to the Euler conditions. Clearly, there may exist deflators $\left(\gamma_{\xi}\right)_{\xi \in D}$ satisfying (5)-(7) that are not compatible with the transversality condition (TC) and, therefore, do not coincide with a plan of Kuhn-Tucker multipliers. In fact, that broader set of deflators satisfying (5), (6) and (7), can be obtained by a non-arbitrage argument, as in the two dates model by Araujo, Fajardo and Páscoa (2005). However, if we pick agent $h$ Kuhn-Tucker multipliers, it follows that non-linearities on asset prices can only arise as a consequence of binding collateral constraints (or, in other words, binding sign constraints on the autonomous consumption, determining positive shadow prices that are responsible for the strict inequality in (5)).

Under monotonicity of preferences, inequalities (6) and (7) are financial non-arbitrage conditions. Thus, by analogy to Magill and Quinzzi (1996) or Santos and Woodford (1997), for some readers it might seem natural to use these two conditions only to analyze the existence of rational asset pricing bubbles. However, since in our model assets are real and commodities may be infinitely durable, we need to understand the asymptotic behavior of commodity prices. To do this, we must also consider inequality (5). Note that in this condition the non-pecuniary returns, $\left(\alpha_{\xi, l}\right)_{l \in L}$, are not vague concepts and can actually be related to marginal utility gains of some agent (by Proposition 
$1(\mathrm{i}))$.

Definition 3. A plan $\Gamma:=\left(\gamma_{\xi}\right)_{\xi \in D} \in \mathbb{R}_{++}^{D}$ is a process of valuation coefficients at prices $(p, q) \in \mathbb{P}$ if there is, for each $\xi \in D$, a vector $\left(\alpha_{\xi, l}\right)_{l \in L} \in \mathbb{R}_{++}^{L}$ such that inequalities (5)-(7) hold.

Thus, any plan of Kuhn-Tucker multipliers of an agent $h$, denoted by $\Gamma^{h}=\left(\gamma_{\xi}^{h}\right)_{\xi \in D}$, is a process of valuation coefficients.

For convenience of future notations, given any process $\Gamma$ of valuation coefficients, for each $\xi \in D$, let $\eta(\Gamma, \xi)=\left(\eta_{x}(\Gamma, \xi, l) ; \eta_{\theta}(\Gamma, \xi, j) ; \eta_{\varphi}(\Gamma, \xi, j)\right)_{(l, j) \in L \times J}$ be the vector defined by

$$
\begin{aligned}
\eta_{x}(\Gamma, \xi, l) & =\gamma_{\xi} p_{\xi, l}-\sum_{\mu \in \xi^{+}} \gamma_{\mu} p_{\mu} Y_{\mu}(\cdot, l)-\alpha_{\xi, l} \\
\eta_{\theta}(\Gamma, \xi, j) & =\gamma_{\xi} q_{\xi, j}-\sum_{\mu \in \xi^{+}} \gamma_{\mu} D_{\mu, j}(p, q) \\
\eta_{\varphi}(\Gamma, \xi, j) & =\gamma_{\xi}\left(p_{\xi} C_{\xi, j}-q_{\xi, j}\right)-\sum_{\mu \in \xi^{+}} \gamma_{\mu}\left(p_{\mu} Y_{\mu} C_{\xi, j}-D_{\mu, j}(p, q)\right)-\alpha_{\xi} C_{\xi, j} .
\end{aligned}
$$

Note that, when $\Gamma=\Gamma^{h}$, for some agent $h \in H, \eta\left(\Gamma^{h}, \xi\right)$ is the vector of shadow prices associated with the collateral constraints and the sign constraints on long and short positions, respectively. Finally, it is important to remark that equation (7) and Assumption B imply that, at each $\xi \in D$,

$$
p_{\xi} C_{\xi, j}>q_{\xi, j}, \quad \forall j \in J
$$

Thus, the collateral cost must exceed the asset price. This condition will be crucial in relating the occurrence of asset price bubbles to the asymptotic behavior of commodity prices.

\section{Equilibrium Existence}

As we point out earlier, when assets live more than one period and agents are infinite lived, three difficulties came up in the literature on equilibrium in default-free economies that made the authors assert only the generic existence of equilibrium and for debt-constrained (or transversality constrained) portfolio plans (as in Hernandez and Santos (1996) or Magill and Quinzii (1996)). ${ }^{6}$ First, when assets live several periods, the rank of the returns matrix will depend on asset prices and, therefore, unless short-sales are bounded, equilibrium existed, in the default-free model, only for a generic set of economies. Second, Ponzi schemes could occur, if debt or transversality restrictions were not imposed. Third, as Hernandez and Santos (1996) pointed out, when asset return matrices are not bounded along the event-tree, equilibria might not exist when infinite-lived real assets are in zero net supply. ${ }^{7}$

However, when assets are collateralized, these difficulties are avoided.

\footnotetext{
${ }^{6}$ Hernandez and Santos (1996) were also able to show the existence of equilibrium in the special case where the asset structure consists of a single infinite lived real asset in positive net supply.

${ }^{7}$ In fact, the asset price can be shown to be the series of discounted real returns and would be unbounded, unless marginal rates of substitution tend to zero quickly enough (which would be the case if the asset's net supply were positive, inducing unbounded additional resources).
} 
Theorem 1. Under Assumptions $A$ and $B$ there exists an equilibrium.

Note that, even in the case of single period assets (see Geanakoplos and Zame (2002)), collateral circumvented the problems associated to the price-dependence of the rank of the return matrices. In fact, collateral is scarce in equilibrium and, therefore, we will have a natural (endogenous) short-sales constraint. Moreover, collateral rules out Ponzi schemes, as it did in the case of single-period assets (see Araujo, Páscoa and Torres-Martínez (2002)). Finally, the existence of collateral guarantees dispenses with any uniform bounds on assets' promised returns, as the asset price is bounded by the discounted value of the depreciated collateral at the next date, plus perhaps some shadow price of the collateral constraint.

\section{Speculative Bubbles in Prices}

As in Magill and Quinzii (1996) and Santos and Woodford (1997), speculation is defined as a deviation of the equilibrium price from the fundamental value of the asset, which is the deflated value of future payments and services that the asset yields. We define fundamental values as a function of the chosen vector of valuation coefficients.

To simplify our analysis, we suppose that, if a commodity consumed at $\xi$ is transforming itself into other goods at the immediate successors nodes of $\xi$, then these goods are one-period perishable.

Assumption C. Given $(\mu, l) \in\left(D \backslash\left\{\xi_{0}\right\}\right) \times L$, if there is $l^{\prime} \neq l$ such that $Y_{\mu}\left(l^{\prime}, l\right) \neq 0$, then at $\mu$ the commodity $l^{\prime}$ is one-period perishable, in the sense that $Y_{\eta}\left(\cdot, l^{\prime}\right)=0, \forall \eta \in \mu^{+}$.

Essentially, this restriction guarantees that fundamental values of commodities may be easily defined in terms of future payments and rental services. Otherwise, the value of payments generated by a good may include speculative terms, induced by the transformation of the good into a durable commodity that has a price bubble.

Speculation in durable goods. The fundamental value at $\xi \in D$ of any commodity $l \in L$ takes into account both the rental services that will be generated in $D(\xi)$ jointly with the payments that will be delivered when $l$ is transformed into another goods.

More formally, given prices $(p, q) \in \mathbb{P}$ and a process of valuation coefficients $\Gamma$, the rental services that $a$ units of commodity $l$ generate at a node $\mu \in D(\xi)$ are given by $\left(p_{\mu, l}-\sum_{\nu \in \mu^{+}} \frac{\gamma_{\nu}}{\gamma_{\mu}} p_{\nu} Y_{\nu}(\cdot, l)\right) a$. On the other hand, the payments that an agent that holds $b$ units of commodity $l$ at $\mu^{-}$receives at node $\mu>\xi$ are given by $\sum_{l^{\prime} \neq l} p_{\mu, l^{\prime}} Y_{\mu}\left(l^{\prime}, l\right) b$.

As commodities only deliver perishable goods (see Assumption $\mathrm{C}$ ), one unit of good $l \in L$ at $\xi \in D$ is transforming into $a_{l}(\xi, \mu)$ units of the same commodity at a node $\mu \in D(\xi)$, where

$$
a_{l}(\xi, \mu)= \begin{cases}\prod_{\xi<\eta \leq \mu} Y_{\eta}(l, l) & \text { if } \mu>\xi \\ 1 & \text { otherwise }\end{cases}
$$


Therefore, under Assumption $\mathrm{C}$ and for a process of valuation coefficients $\Gamma$, the fundamental value of commodity $l$ at node $\xi$ is defined by

$F_{l}(\xi, p, q, \Gamma)=\sum_{\mu \in D(\xi)} \frac{\gamma_{\mu}}{\gamma_{\xi}}\left(p_{\mu, l}-\sum_{\nu \in \mu^{+}} \frac{\gamma_{\nu}}{\gamma_{\mu}} p_{\nu} Y_{\nu}(\cdot, l)\right) a_{l}(\xi, \mu)+\sum_{\mu>\xi} \frac{\gamma_{\mu}}{\gamma_{\xi}} \sum_{l^{\prime} \neq l} p_{\mu, l^{\prime}} Y_{\mu}\left(l^{\prime}, l\right) a_{l}\left(\xi, \mu^{-}\right)$.

Furthermore, for each $T>t(\xi)$,

$$
\begin{array}{r}
p_{\xi, l}=\sum_{\mu \in D^{T}(\xi)} \frac{\gamma_{\mu}}{\gamma_{\xi}}\left(p_{\mu, l}-\sum_{\nu \in \mu^{+}} \frac{\gamma_{\nu}}{\gamma_{\mu}} p_{\nu} Y_{\nu}(\cdot, l)\right) a_{l}(\xi, \mu)+\sum_{\mu \in D^{T}(\xi) \backslash\{\xi\}} \frac{\gamma_{\mu}}{\gamma_{\xi}} \sum_{l^{\prime} \neq l} p_{\mu, l^{\prime}} Y_{\mu}\left(l^{\prime}, l\right) a_{l}\left(\xi, \mu^{-}\right) \\
+\sum_{\mu \in D_{T+1}(\xi)} \frac{\gamma_{\mu}}{\gamma_{\xi}} p_{\mu} Y_{\mu}(\cdot, l) a_{l}\left(\xi, \mu^{-}\right) .
\end{array}
$$

Since, independently of $T$, the last term on the right hand side of the equation above is nonnegative, it follows that, for any choice of $\Gamma$ the fundamental value of commodity $l$ is well defined and less than or equal to the unitary price. Also, taking the limit as $T$ goes to infinity, we conclude that, $p_{\xi, l}=F_{l}(\xi, p, q, \Gamma)+\lim _{T \rightarrow+\infty} \sum_{\mu \in D_{T}(\xi)} \frac{\gamma_{\mu}}{\gamma_{\xi}} p_{\mu} Y_{\mu}(\cdot, l) a_{l}\left(\xi, \mu^{-}\right)$.

DeFINITION 3. Given a process $\Gamma$ of valuation coefficients, we say that the price of commodity $l \in L$ has a $\Gamma$-bubble at node $\xi$ when $p_{\xi, l}>F_{l}(\xi, p, q, \Gamma)$.

\section{Characterization of BUbBles ON COMMODity PRICES.}

There is a $\Gamma$-bubble on commodity $l \in L$ price at node $\xi \in D$ if and only if

$$
\lim _{T \rightarrow+\infty} \sum_{\mu \in D_{T}(\xi)} \frac{\gamma_{\mu}}{\gamma_{\xi}} p_{\mu} Y_{\mu}(\cdot, l) a_{l}\left(\xi, \mu^{-}\right)>0
$$

A commodity $l$ has finite durability at a node $\xi$ if there exists $N>0$ such that $a_{l}(\xi, \mu)=0$ for all $\mu \in D(\xi) \backslash D^{N}(\xi)$. It follows from the characterization above that, under Assumption C, commodities with finite durability at $\xi$ are free of bubbles. ${ }^{8}$ For commodities with infinite durability, sufficient conditions for the absence of bubbles are given by the next result.

TheOrem 2. Given equilibrium prices $(p, q) \in \mathbb{P}$, suppose that Assumptions $A, B$ and $C$ hold. $A$ sufficient condition for commodities to be free of $\Gamma$-bubbles in $D(\xi)$ is that,

$$
\exists h \in H, \quad \sum_{\mu \in D(\xi)} \frac{\gamma_{\mu}}{\gamma_{\xi}} p_{\mu} W_{\mu}^{h}<+\infty .
$$

Given $h \in H$, commodities are free of $\Gamma^{h}$-bubbles in $D(\xi)$ if any of the following conditions hold,

(i) At any node, agent $h$ receives at least a fraction $k \in(0,1)$ of aggregated endowments. That is, $\kappa W_{\mu}^{h} \leq w_{\mu}^{h}$ for all $\mu \in D(\xi)$.

(ii) Cumulated depreciation factors $Y_{\xi, \mu}$ are uniformly bounded by above in $D(\xi)$ and new endowments, $\left(w_{\mu}^{h}\right)_{\mu \in D(\xi)}$, are uniformly bounded away from zero in $D(\xi)$.

\footnotetext{
${ }^{8}$ When Assumption $\mathrm{C}$ is not satisfied, even commodities with finite durability may have bubbles, as may transform into other goods with infinite durability whose prices have bubbles.
} 
(iii) Individuals' inter-temporal marginal rates of substitution coincide along the event-tree, i.e., given $h^{\prime} \in H$ there is $\pi>0$ such that, $\left(\pi \gamma_{\xi}^{h}\right)_{\xi \in D}$ is a plan of Kuhn-Tucker multipliers for $h^{\prime}$.

Proof. Fix $\eta \geq \xi$ and $l \in L$. Assume that condition (9) holds. It follows by Assumption A that, for each $T>t(\eta)$,

$$
\sum_{\mu \in D_{T}(\eta)} \frac{\gamma_{\mu}}{\gamma_{\eta}} p_{\mu} Y_{\mu}(\cdot, l) a_{l}\left(\eta, \mu^{-}\right) \leq \frac{1}{W_{\eta, l}^{h}} \sum_{\mu \in D_{T}(\eta)} \frac{\gamma_{\mu}}{\gamma_{\eta}} p_{\mu} W_{\mu}^{h} .
$$

Taking the limit as $T$ goes to infinity, we conclude that $p_{\eta, l}$ is free of $\Gamma$-bubbles.

Given $h \in H$, if (i) holds, it follows from item (iii) of Proposition 1 that condition (9) is satisfied, that concludes the proof. Also, if (ii) is satisfied, item (iii) of Proposition 1 assure that bubbles are ruled out. Finally, suppose that equilibrium individual marginal rates of substitution coincide along the event-tree. Then, transversality conditions $\left(\mathrm{TC}_{x}\right),\left(\mathrm{TC}_{\varphi}\right)$ and $\left(\mathrm{TC}_{\theta}\right)$ hold, for all agents under a same deflator. Adding up these three conditions across all agents, we get condition (9) above.

Condition (iii) in the above theorem requires the processes of individuals' Kuhn-Tucker multipliers to be collinear. It is well known that in unrestricted financial markets, this condition is a characteristic property of complete markets and, therefore, equivalent to the property that the rank of the matrix of returns of non-redundant assets should be equal to the number of immediate successor nodes. However, in the presence of binding financial constraints this equivalence may no longer hold. Giménez (2003) made this point in the context of short-sales constraints and gave examples of equilibrium where the above returns matrix had full rank but the presence of a shadow price for these constraints led to multiplicity of multipliers for each agent and non-collinear multipliers across agents. The markets illustrated in those examples were referred by Giménez (2003) as technically incomplete, along the lines of an earlier discussion done by Santos and Woodford (1992, 1996). In our context, the collateral constraint might be binding as well and if the respective shadow price were non-zero, the uniqueness of the Kuhn-Tucker multipliers process would no longer be guaranteed by a full-rank property of the returns matrix. Hence, our condition (iii) requires more than just that full-rank property, it requires completeness in the stricter sense proposed by Giménez (2003) for asset-constrained economies.

Asset Pricing Bubbles. The fundamental value of an asset is the present value of its future yields and services. Future yields are the perishable goods directly or indirectly delivered by the asset. Real payments of perishable commodities are the yields directly delivered. Indirect delivered yields are the perishable commodities obtained by the transformations of real payments into other goods, or by the transformation of these goods into others and so on. Future services include the shadow prices of the financial constraints and the rental values of the delivered durable goods. These goods are received as an original promise or as a collateral garnishment, and are unambiguously anticipated except in the borderline case, when the value of the promise equals the garnishable collateral value. Thus, the fundamental value will depend not just on the process of valuation coefficients but also on the believed delivery rates for the borderline nodes. 
Also, there is no reason to pick the equilibrium delivery rates instead of any other rates that may treat differently the deliveries only in the borderline case. That is, for purposes of valuation, anticipating that agents pay the promise or anticipating that they surrender the garnishable collateral (or a convex combination of the two) are equally perfectly acceptable when agents are indifferent between these two actions. Recall that each agent does not care about this choice and does not know what are the other agents' choices.

Given equilibrium prices $(p, q)$ we define fundamental values in terms of any believed delivery rates compatible with individual rationality, that is, any process $\tau=\left(\tau_{\xi, j}\right) \in[0,1]^{\left(D \backslash\left\{\xi_{0}\right\}\right) \times J}$, such that,

$$
\tau_{\xi, j}= \begin{cases}1 & \text { if } p_{\xi} A(\xi, j)+q_{\xi, j}<p_{\xi} \widehat{C}_{\xi, j} \\ 0 & \text { if } p_{\xi} A(\xi, j)+q_{\xi, j}>p_{\xi} \widehat{C}_{\xi, j}\end{cases}
$$

Under delivery rates $\tau$, the physical bundle that one unit of asset $j$ negotiated at node $\xi$ delivers at $\mu \in \xi^{+}$, namely $P D_{\mu, j}(\tau)$, consists of the part of the promises $A_{\mu, j}$ that are effectively delivered and also of the physical deliveries made by the garnished collateral. More precisely, $P D_{\mu, j}(\tau)=$ $\tau_{\mu, j} A_{\mu, j}+\left(1-\tau_{\mu, j}\right) \widehat{C}_{\xi, j}$.

Thus, we have that, $D_{\mu, j}(p, q)=p_{\mu} P D_{\mu, j}(\tau)+\tau_{\mu, j} q_{\mu, j}$. Using inequality (6) we obtain that,

$$
\begin{aligned}
q_{\xi, j} & =\sum_{\mu \in \xi^{+}} \frac{\gamma_{\mu}}{\gamma_{\xi}}\left(p_{\mu} P D_{\mu, j}(\tau)+\tau_{\mu, j} q_{\mu, j}\right)+\frac{\eta_{\theta}(\Gamma, \xi, j)}{\gamma_{\xi}} \\
& =\sum_{\mu>\xi}\left(\prod_{\xi<\eta<\mu} \tau_{\eta, j}\right)\left(\frac{\gamma_{\mu}}{\gamma_{\xi}} p_{\mu} P D_{\mu, j}(\tau)+\frac{\eta_{\theta}\left(\Gamma, \mu^{-}, j\right)}{\gamma_{\xi}}\right)+\lim _{T \rightarrow+\infty} \sum_{\mu \in D_{T}(\xi)} \frac{\gamma_{\mu}}{\gamma_{\xi}} q_{\mu, j} \prod_{\xi<\eta \leq \mu} \tau_{\eta, j} .
\end{aligned}
$$

Under $(\Gamma, \tau)$, the fundamental value of an asset $j \in J$ at a node $\xi, F_{j}(\xi, p, q, \Gamma, \tau)$, is defined by

$$
F_{j}(\xi, p, q, \Gamma, \tau)=\sum_{\mu>\xi}\left(\prod_{\xi<\eta<\mu} \tau_{\eta, j}\right)\left[\frac{\gamma_{\mu}}{\gamma_{\xi}} \sum_{l \in L} F_{l}(\mu, p, q, \Gamma) P D_{\mu, j}(\tau)_{l}+\frac{\eta_{\theta}\left(\mu^{-}, j\right)}{\gamma_{\xi}}\right]
$$

It follows that, independently of $(\Gamma, \tau)$, the fundamental value at $\xi$ is always well defined and less than or equal to the unitary asset price, $q_{\xi, j}$.

Definition 4. Given equilibrium prices $(p, q) \in \mathbb{P}$, we say that the price of asset $j \in J$ has a $(\Gamma, \tau)$-bubble at a node $\xi$ when $q_{\xi, j}>F_{j}(\xi, p, q, \Gamma, \tau)$.

By definition, a bubble on asset $j$ may be a consequence of a bubble in a commodity — used as collateral or that is part of the real promises - or may be generated by asymptotically positive asset prices. As assets are backed by physical collateral, the non-arbitrage condition given by equation (8) allows us to find a relationship between the asymptotic value of asset prices and the asymptotic value of collateral bundles. For this reason, and differently from what happens in models without default, the existence of bubbles in financial markets is strongly related to the existence of speculation in physical markets. 
Theorem 3. Given equilibrium prices $(p, q) \in \mathbb{P}$, suppose that Assumptions $A, B$ and $C$ hold. The price of asset $j \in J$ is free of $(\Gamma, \tau)$-bubbles in $D(\xi)$ if the following conditions hold,

$$
\exists h \in H: \quad \sum_{\mu \in D(\xi)} \frac{\gamma_{\mu}}{\gamma_{\xi}} p_{\mu} W_{\mu}^{h}<+\infty, \quad \text { and } \quad \lim _{T \rightarrow+\infty} \sum_{\mu \in D_{T}(\xi)} \frac{\gamma_{\mu}}{\gamma_{\xi}} q_{\mu, j}=0 .
$$

Given $h \in H$, asset $j$ 's price is free of $\left(\Gamma^{h}, \tau\right)$-bubbles in $D(\xi)$ if any of the following conditions hold,

(i) Commodities are free of $\Gamma$-bubbles in $D(\xi)$ and asset $j$ is a mortgage loan in $D(\xi)$, i.e., $C_{\mu, j} \leq Y_{\xi, \mu} C_{\xi, j}$, for any $(\mu, l) \in(D(\xi) \backslash\{\xi\}) \times L$.

(ii) Collateral requirements $\left(C_{\mu, j}\right)_{\mu \in D(\xi)}$ are uniformly bounded, cumulated depreciation factors $Y_{\xi, \mu}$ are uniformly bounded by above in $D(\xi)$ and $\left(w_{\mu}^{h}\right)_{\mu \in D(\xi)}$ is uniformly bounded away from zero in $D(\xi)$.

(iii) Net supply of asset $j$ is persistently positive and there are Kuhn-Tucker multipliers for the other agents in $H$ which are collinear with $\Gamma^{h}$.

Proof. Under the conditions of item (i), asset $j$ has a $(\Gamma, \tau)$-bubble at a node $\eta \geq \xi$ only if $\lim _{T \rightarrow+\infty} \sum_{\mu \in D_{T}(\eta)} \frac{\gamma_{\mu}}{\gamma_{\eta}} q_{\mu, j}>0$. This is incompatible with the absence of commodity bubbles in $D(\xi)$. In fact, using the non-arbitrage condition (8), Assumption $\mathrm{C}$ together with the particular collateral structure of the mortgage imply that $q_{\mu, j} \leq p_{\mu} C_{\mu, j} \leq \sum_{l \in L} p_{\mu, l} Y_{\mu}(\cdot, l) a_{l}\left(\mu^{-}, \xi\right) C_{\xi, j, l}$.

Assume that the hypotheses of item (ii) holds. It follows from Theorem 2 that commodities are free of bubbles in $D(\xi)$. Since $\left(w_{\mu}^{h}\right)_{\mu \geq \xi}$ is uniformly bounded away from zero, it follows from item (iii) in Proposition 1 that, for any $\eta \in D(\xi), \sum_{\eta \in D} \frac{\gamma_{\mu}}{\gamma_{\eta}^{h}}\left\|p_{\mu}\right\|_{\Sigma}<+\infty$. Thus, independently of $\tau$, assets are free of $\left(\Gamma^{h}, \tau\right)$-bubbles due that collateral requirements are uniformly bounded and condition (8) holds.

Under the conditions of item (iii), let $e_{\mu}^{j}$ be the aggregate net supply of an asset $j$ at node $\mu \geq \xi$. It follows from $\left(\mathrm{TC}_{\theta}\right)$, using the financial feasibility condition that, for each $\eta \geq \xi$, $\lim _{T \rightarrow+\infty} \sum_{\mu \in D_{T}(\eta)} \frac{\gamma_{\mu}^{h}}{\gamma_{\eta}^{h}} \sum_{j \in J} q_{\mu, j} e_{\mu}^{j}=0$. Therefore, as $\left(e_{\mu}^{j}\right)_{\mu \geq \eta}$ is bounded away from zero in $D(\eta)$, we have that $\lim _{T \rightarrow+\infty} \sum_{\mu \in D_{T}(\eta)} \frac{\gamma_{\mu}^{h}}{\gamma_{\eta}^{h}} q_{\mu, j}=0$. Also, commodities do not have bubbles in $D(\eta)$ (see item (iii) of Theorem 2). Therefore, asset $j$ is free of bubbles at $\eta \in D(\xi)$.

It follows from item (i) above that, a bubble in a mortgage loan is always a consequence of a bubble in a commodity that is used as collateral or is part of the real promises. On the other hand, when commodities neither appreciate nor transform into other goods along the event-tree, it follows from item (ii) that, under bounded unitary collateral requirements, well behaved initial endowments assure the absence of price bubbles. In fact, assets will not have a positive price at infinity if the sequence of deflated asset prices is summable, but as this sequence is dominated by the sequence of deflated collateral costs (by non-arbitrage), we just need to have collateral coefficients to be uniformly bounded and deflated commodity prices to be summable (which follows by what is assumed on endowments and depreciation matrices). 
In a straightforward extension of our model, we could have allowed for finite-lived assets and show that price bubbles would occur if the commodities serving as collateral are priced at infinity. Indeed, the price of a finitely-lived asset will have a bubble if the asset pays in durable goods whose prices have bubbles or if the asset defaults and the surrendered physical collateral is subject to price bubbles.

\section{Examples of Bubbles}

In our first example, the single asset is persistently in positive net supply, but it has a price bubble under Kuhn-Tucker deflators, which yield finite present values of wealth. Essentially, differently from the conditions in item (iii) of Theorem 3, individuals' Kuhn-Tucker deflators are not collinear.

Example 1. Each $\xi \in D$ has two successors: $\xi^{+}=\left\{\xi^{u}, \xi^{d}\right\}$. There are two commodities and a single infinite-lived asset. The first commodity is a perishable good $x_{1}$ and the second one is a durable good $x_{2}$, which is subject to a constant rate of depreciation $\kappa \in(0,1)$. Commodity prices at node $\xi$ are denoted by $\left(p_{\xi, 1}, p_{\xi, 2}\right)$. The asset, money, pays no dividend and its short-sales have to be collateralized with coefficients $C_{\xi}=(0,2)$. Thus, at any node $\xi$, the asset's effective payments become $\min \left\{q_{\xi}, 2 \kappa p_{\xi, 2}\right\}$, where $q_{\xi}$ denotes the price of money at $\xi$.

There are two agents $h \in H=\{1,2\}$. Each $h \in H$ has physical endowments $\left(w_{\xi, 1}^{h}, w_{\xi, 2}^{h}\right)_{\xi \in D}$, receives financial endowments $e^{h} \geq 0$ only at the first node, and has preferences represented by the utility function $U^{h}(\widehat{x})=\sum_{\xi \in D} \beta^{t(\xi)} \rho^{h}(\xi)\left(\widehat{x}_{\xi, 1}+\sqrt{\widehat{x}_{\xi, 2}}\right)$, where $\beta \in(0,1)$ and $\rho^{h}(\xi) \in(0,1)$ satisfies $\rho\left(\xi_{0}\right)=1, \rho^{h}(\xi)=\rho^{h}\left(\xi^{d}\right)+\rho^{h}\left(\xi^{u}\right), \forall \xi \in D$.

The depreciation factor is such that $\kappa<\delta:=\beta^{2}$ and, for any $\xi \in D$,

$$
\rho^{1}\left(\xi^{u}\right)=\frac{1}{2^{t(\xi)+1}} \rho^{1}(\xi), \quad \rho^{2}\left(\xi^{u}\right)=\left(1-\frac{1}{2^{t(\xi)+1}}\right) \rho^{2}(\xi) .
$$

Let $D^{d u}$ be the set of nodes attained after going down followed by up, that is, $D^{d u}=\{\eta \in D$ : $\left.\exists \xi, \quad t(\eta)=t(\xi)+2 \wedge \eta=\left(\xi^{d}\right)^{u}\right\}$ and let $D^{u d}$ be the set of nodes reached by going up and then down, that is, $D^{u d}=\left\{\eta \in D: \exists \xi, t(\eta)=t(\xi)+2 \wedge \eta=\left(\xi^{u}\right)^{d}\right\}$.

Agent $h=1$ is the only one endowed with the asset, i.e. $\left(e^{1}, e^{2}\right)=(e, 0)$. Physical endowments at initial node are $w_{\xi_{0}, 1}^{h}=w_{\xi_{0}, 2}^{h}=1$. Moreover, for each $\xi \neq \xi_{0}$, define $w_{\xi, 2}^{h}=\delta^{t(\xi)}-\kappa \delta^{t(\xi)-1}$ and $w_{\xi, 1}^{h}=1+d_{\xi}^{h}$, where

$$
d_{\xi}^{1}=\left\{\begin{array}{ll}
\frac{\kappa e}{2(1-\kappa)} \beta^{-t(\xi)}, & \text { if } \xi \in D^{d u} ; \\
0, & \text { otherwise. }
\end{array} \quad d_{\xi}^{2}= \begin{cases}\frac{\kappa e}{2(1-\kappa)} \beta^{-t(\xi)}, & \text { if } \xi \in\left\{\xi_{0}^{d}\right\} \cup D^{u d} \\
0, & \text { otherwise. }\end{cases}\right.
$$

Notice that the cumulated endowments of the durable good are tending to zero at the rate $\beta^{2}$ and, therefore, given the specific form of the marginal utility, the scarcity of this commodity tends to offset the discount factor and, in equilibrium, each agent should consume the own cumulated endowment. But the shocks on endowments of the perishable good create an opportunity to use money as a way to transfer wealth to states that are more valuable.

Since agents receive positive shocks in states that are assigned low probabilities, there is an incentive to use these positive shocks to buy money and sell it later in states with higher probabilities. Thus, we will look for an equilibrium where agent $h=1$, the one that starts endowed with money, 
gets rid of it when going down (to which she attaches a higher conditional probability), but, if afterwards she goes up, she will use the positive perishable endowment shock to buy back money (and be back in a financial position analogous to the one she had at the original node). Clearly, each agent should end up consuming the other agent's positive shock.

Let $\left(p_{\xi, 1}, p_{\xi, 2}, q_{\xi}\right)_{\xi \in D}=\left(2(1-\kappa) \beta^{t(\xi)}, 1, \kappa\right)_{\xi \in D}$. For these prices, default never occurs (as $q_{\xi}<$ $\left.2 \kappa p_{\xi, 2}\right)$ and effective asset payments are given by $q_{\xi}$. Suppose that $\left(\widehat{x}_{\xi, 1}^{h}, \widehat{x}_{\xi, 2}^{h}\right)=\left(1+d_{\xi}^{h^{\prime}}, \delta^{t(\xi)}\right)$, where $h \neq h^{\prime}$. Thus, it follows from budget constraints that, at each $\xi$, the portfolio of agent $h$ must satisfy $z_{\xi}^{h}=2 \frac{1-\kappa}{\kappa} \beta^{t(\xi)}\left(d_{\xi}^{h}-d_{\xi}^{h^{\prime}}\right)+z_{\xi^{-}}^{h}$, where $z_{\xi_{0}^{-}}^{h}:=e^{h}$.

Consumption allocations above jointly with the portfolios $\left(z_{\xi_{0}}^{1}, z_{\xi^{u}}^{1}, z_{\xi^{d}}^{1}\right)=(e, e, 0)$ and $\left(z_{\xi}^{2}\right)_{\xi \in D}=$ $\left(e-z_{\xi}^{1}\right)_{\xi \in D}$ are budget feasible. As there are no short sales, collateral constraints are never binding. Moreover, physical and financial market clearing hold.

Finally, if we choose, for each $(h, \xi) \in H \times D, \gamma_{\xi}^{h}=\rho^{h}(\xi) \frac{1}{2(1-\kappa)}$, the following Euler and transversality conditions hold and they are sufficient for individual optimality (see Proposition 1 ),

$$
\begin{aligned}
& \gamma_{\xi}^{h} p_{\xi, x}=u_{\xi, x}^{\prime}\left(1+d_{\xi}^{h^{\prime}}, \delta^{t(\xi)}\right) \\
& \gamma_{\xi}^{h}=\kappa\left(\gamma_{\xi^{u}}^{h}+\gamma_{\xi^{d}}^{h}\right)+u_{\xi, y}^{\prime}\left(1+d_{\xi}^{h^{\prime}}, \delta^{t(\xi)}\right) \\
& \gamma_{\xi}^{h} q_{\xi}=\gamma_{\xi^{u}}^{h} q_{\xi^{u}}+\gamma_{\xi^{d}}^{h} q_{\xi^{d}} \\
& \sum_{\eta \in D_{T}} \gamma_{\eta}^{h}\left(p_{\eta, x} \widehat{x}_{\eta, 1}^{h}+\widehat{x}_{\eta, 2}^{h}\right)+\sum_{\eta \in D_{T}} \gamma_{\eta}^{h} q_{\eta} \theta_{\eta}^{h} \longrightarrow 0, \quad \text { as } T \rightarrow+\infty .
\end{aligned}
$$

In equilibrium, money has an unambiguous bubble, since collateral constraints never bind (which implies that shadow prices of financial constraints are zero) and, therefore, the fundamental value of money is zero. Also, both agents perceive finite present values of aggregate wealth. Indeed, aggregated endowments up to node $\xi$ are $W_{\xi}=W_{\xi}^{1}+W_{\xi}^{2}=\left(2+\sum_{h} d_{\xi}^{h}, 2 \delta^{t(\xi)}\right)$ and, therefore, given $\xi \in D$, for each $h \in\{1,2\}$,

$$
\sum_{\mu \geq \xi} \frac{\gamma_{\mu}^{h}}{\gamma_{\xi}^{h}} p_{\mu} W_{\mu} \leq \frac{1}{\rho^{h}(\xi)}\left(4 \frac{1-\kappa}{1-\beta}+2 \frac{1}{1-\delta}+\kappa e \sum_{\mu \in D^{u d} \cup D^{d u} \cup\left\{\xi_{0}^{d}\right\}} \rho^{h}(\mu)\right),
$$

where

$$
\begin{aligned}
\sum_{\mu \in D^{u d} \cup D^{d u}} \rho^{h}(\mu) & \leq \sum_{t \geq 0} \sum_{\mu \in D_{t+2} \cap\left(D^{u d} \cup D^{d u}\right)} \rho^{h}(\mu) \\
& =\sum_{t \geq 0} \sum_{\mu \in D_{t}}\left(\frac{1}{2^{t+1}}\left(1-\frac{1}{2^{t+2}}\right)+\frac{1}{2^{t+2}}\left(1-\frac{1}{2^{t+1}}\right)\right) \rho^{h}(\mu)<\frac{5}{3} .
\end{aligned}
$$

It is important to understand why the above bubble in the price of an asset in positive net supply is compatible with the transversality conditions that are necessary for individual optimality. The point is that agents do not agree in not lending at infinity under a same process of multipliers $\left(\gamma_{\xi}^{h}\right)_{\xi \in D}$. For instance, agent $h=1$ is a lender at infinity when the future is discounted using agent 2's Kuhn-Tucker multipliers,

$$
\sum_{\eta \in D_{T}} \gamma_{\eta}^{2} q_{\eta} \theta_{\eta}^{1}=\kappa e \sum_{\left\{\eta \in D_{T}: \eta=\left(\eta^{-}\right)^{u}\right\}} \gamma_{\eta}^{2}=\frac{\kappa e}{2(1-\kappa)}\left(1-\frac{1}{2^{T}}\right) \longrightarrow_{T \rightarrow+\infty} \frac{\kappa e}{2(1-\kappa)} .
$$


Our second example illustrates the endogenous reduction (due to collateral seizure) in the net supply of an asset. In this example, the asset is again money and it has also a positive price.

Example 2. Consider a deterministic economy with two infinite lived agents, $h \in\{1,2\}$, one durable good and one long lived asset, money. There are asset endowments, $e^{h}$, only at the original date. Physical endowments are given by $w_{t}^{h}$. Let $\widehat{x}_{t}^{h}$ be the consumption choices of agent $h$. Agents preferences are given by $U^{h}\left(\widehat{x}_{0}, \widehat{x}_{1}, \ldots\right)=\sum_{t=0}^{+\infty} \beta^{t} \sqrt{\widehat{x}_{t}}$, where $\beta \in(0,1)$. We take the commodity as the numeraire. The physical collateral coefficient at date $t$ is $C_{t}=1$, and the commodity depreciation rate is also constant, given by $\kappa \in(0,1)$. Now, let $\delta=\beta^{2}$ and take $\kappa \in\left(\frac{\delta}{2}, \delta\right)$. Suppose that individual endowments are given by,

$$
\begin{aligned}
& w_{0}^{h}=1+(-1)^{h+1} \kappa s_{0}, \\
& w_{t}^{h}=\delta^{t}-\kappa \delta^{t-1}-\kappa \alpha(1-\alpha)^{t-1}\left(e^{h}+(-1)^{h+1} s_{0}\right), \quad \forall t>0,
\end{aligned}
$$

where, for non-negativity of endowments, we require that

$$
\alpha \in\left(\frac{\delta-\kappa}{\kappa}, 1\right), \quad \delta>1-\alpha, \quad s_{0}<\frac{\delta-\kappa}{\kappa \alpha}-\max _{h} e^{h}, \quad e^{h}<\frac{\delta-\kappa}{\kappa \alpha} .
$$

Take, for example, $\left(\beta, \delta, \kappa, \alpha, s_{0}, e^{1}, e^{2}\right)=\left(\frac{\sqrt{2}}{2}, \frac{1}{2}, \frac{1}{3}, \frac{3}{4}, \frac{1}{8}, \frac{1}{2}, 0\right)$.

Denote agent $h$ portfolio by $z_{t}^{h}$. At each date $t>0$, the asset's effective nominal return is $R_{t}=\min \left\{q_{t}, \kappa\right\}$. We can write $R_{t}=\left(1-\lambda_{t}\right) \kappa+\lambda_{t} q_{t}$, where the delivery rate satisfies $\lambda_{t}=1$ if $\kappa>q_{t}$ and $\lambda_{t}=0$ if $\kappa<q_{t}$.

The collateral constraint can be written as $\widehat{x}_{t}^{h} \geq-z_{t}^{h}$ and the budget constraints, for a nonnegative plan $\left(\widehat{x}_{t}^{h}\right)_{t \geq 0}$, are given by,

$$
\begin{aligned}
\widehat{x}_{0}^{h}+q_{0} z_{0}^{h} & =w_{0}^{h}+q_{0} e_{0}^{h} ; \\
\widehat{x}_{t}^{h}+q_{t} z_{t}^{h} & =w_{t}^{h}+\kappa \widehat{x}_{t-1}^{h}+R_{t} z_{t-1}^{h}, \text { for } t>0 .
\end{aligned}
$$

Market clearing conditions are as defined in Section 2.

We look for an equilibrium without default (that is, where $q_{t} \leq \kappa$, for all $t>0$ ) and with non-binding collateral constraints. By Proposition 1, a budget feasible plan $\left(\widehat{x}_{t}^{h}, z_{t}^{h}\right)_{t \geq 0}$ is optimal for agent $h$ if there exist non-negative multipliers $\gamma_{t}^{h}$ such that, the following Euler equations and transversality conditions hold, ${ }^{9}$ for each $t \geq 0$,

$$
\begin{aligned}
\gamma_{t}^{h} q_{t} & =\gamma_{t+1}^{h} q_{t+1}, \\
\gamma_{t}^{h} & =\kappa \gamma_{t+1}^{h}+\beta^{t} \sqrt{\widehat{x}_{t}^{h}}, \\
0 & =\lim _{t \rightarrow+\infty} \gamma_{t}^{h}\left(\widehat{x}_{t}^{h}+q_{t} z_{t}^{h}\right) .
\end{aligned}
$$

An equilibrium. Let, for all $t \geq 0, \gamma_{t}^{h}=\frac{1}{2(1-\kappa)}$ and $q_{t}=\kappa$ (hence $R_{t}=\kappa$ ). Individual optimal plans are given by $\widehat{x}_{t}^{h}=\delta^{t}$ and $z_{t}^{h}=(1-\alpha)^{t}\left((-1)^{h+1} s_{0}+e^{h}\right)$. Collateral constraints are not binding. Note that the asset is in the borderline case for each $t \geq 1$ and, if we take the delivery rate $\lambda_{t}$ to be

\footnotetext{
${ }^{9}$ Notice that the Euler equations with respect to $\widehat{x}_{t}^{h}$ and $z_{t}^{h}$, conditions (11) and (12), imply the Euler conditions with respect to $\left(x_{t}^{h}, \theta_{t}^{h}, \varphi_{t}^{h}\right)$.
} 
equal to $1-\alpha$, then all market clearing conditions hold at each date.

Now, commodity prices do not have bubbles, since at each date $t \geq 0, \lim _{T \rightarrow+\infty} \frac{\gamma_{T}^{h}}{\gamma_{t}^{h}} p_{t} Y_{t, T}=0$. Moreover, as collateral constraints are not binding, the respective shadow prices are zero and, therefore, if agents believe that in the borderline case the asset pays the original promise, it follows that the fundamental value of money at date $t$ is zero and, as $q_{t}>0$, money has a bubble at each date.

As argued in the previous section, the above belief about the deliveries may diverge from the equilibrium delivery rates. When an agent evaluates whether there is speculation or not in an asset, the agent is concerned with values and therefore it is perfectly reasonable to anticipate full delivery in borderline situations. But other beliefs should also be allowed and the above monetary equilibrium may be reinterpreted as a positive fundamental value (or even as a situation where bubbles and positive fundamental values coexist).

In fact, under delivery beliefs $\left(\tau_{t}\right)_{t \geq 1} \in[0,1]^{\mathbb{N}}$, the fundamental value of money is given by,

$$
F(t, p, q, \Gamma, \tau)=\sum_{t^{\prime}>t}\left(\prod_{t<s<t^{\prime}} \tau_{s}\right)\left(1-\tau_{t^{\prime}}\right) \kappa=\kappa\left(1-\lim _{T \rightarrow+\infty} \prod_{t<s \leq T} \tau_{s}\right),
$$

which implies that the asset has a bubble at date $t$, i.e. $q_{t}=\kappa>F(t, p, q, \Gamma, \tau)$, if and only if $\prod_{t<s \leq T} \tau_{s}$ converges to a strictly positive limit as $T$ goes to infinity.

If the limit above is strictly positive and less than one, the asset has a bubble and at the same time the fundamental value is positive. If the delivery expectations are zero at some node (so that the above limit is trivially zero), then the monetary equilibrium reduces to a positive fundamental value induced by the fundamental value of the commodities that serve as collateral. In this case, the price of money would consist of a positive fundamental value even though shadow prices of the collateral constraints are zero.

The examples above show that collateral allows for price bubbles in assets in positive net supply. This class of assets is quite important, as it includes equity contracts and money. Our examples focus precisely on the latter and in a context without any liquidity frictions. Hence, bubbles have the intriguing feature of assigning a positive price to an asset having no dividends and also providing no services.

Contrary to other approaches where the positive price of money is due to a positive fundamental value that adds up the shadow prices of binding liquidity constraints (see Santos (2006)) or shortsales constraints (see Gimenez (2007)), in our examples there are no binding collateral constraints and the monetary equilibria are either (i) unambiguous money bubbles (Example 1) or (ii) bubbles that can be reinterpreted as positive fundamental values due to collateral seizure (Example 2).

The latter occur under an endogenous reduction in assets' net supply, as the collateral takes the place of the promise, and are a new instance for the long standing view on the efficiency properties resulting from a vanishing money supply (see Friedman (1969), Grandmont and Younés (1972, 1973) or Woodford (1994), among others). The former are compatible with persistent money supply, but can only occur in the case in which individuals' marginal rates of substitution of wealth do not coincide, by taking advantage of the diversity in agents' personalized deflators. 


\section{Appendix A}

Following the notation of Section 4 , given $(p, q) \in \mathbb{P}$, let $\partial v_{\xi}^{h}\left(z_{\xi}\right)$ be the super-differential set of the function $v_{\xi}^{h}$ at the point $z_{\xi}$. Note that, a vector $\left(\mathcal{L}_{\xi, 1}^{\prime}, \mathcal{L}_{\xi, 2}^{\prime}\right) \in \partial \mathcal{L}_{\xi}^{h}\left(z_{\xi}, z_{\xi^{-}}, \gamma ; p, q\right)$ if and only there exists $v_{\xi}^{\prime} \in \partial v_{\xi}^{h}\left(z_{\xi}\right)$ such that both $\mathcal{L}_{\xi, 1}^{\prime}=v_{\xi}^{\prime}-\gamma \nabla_{1} g_{\xi}^{h}(p, q)$ and $\mathcal{L}_{\xi, 2}^{\prime}=-\gamma \nabla_{2} g_{\xi}^{h}(p, q)$, where $\nabla_{1} g_{\xi}^{h}(p, q)=$ $\left(p_{\xi}, q_{\xi}, p_{\xi} C_{\xi, j}-q_{\xi}\right)$ and $\left.\nabla_{2} g_{\xi}^{h}(p, q)\right)=-\left(p_{\xi} Y_{\xi}, D_{\xi}(p, q),\left(p_{\xi} Y_{\xi} C_{\xi^{-}, j}-D_{\xi, j}(p, q)\right)_{j \in J}\right)$. Therefore, for any $\left(\mathcal{L}_{\xi, 1}^{\prime}, \mathcal{L}_{\xi, 2}^{\prime}\right) \in \partial \mathcal{L}_{\xi}^{h}\left(z_{\xi}, z_{\xi^{-}}, \gamma ; p, q\right)$, we have $\mathcal{L}_{\xi, 2}^{\prime} \geq 0$.

Proof of Proposition 1. (i) For any $T \in \mathbb{N}$, consider the truncated optimization problem,

$$
\begin{aligned}
\max & \sum_{\xi \in D^{T}} v_{\xi}^{h}\left(z_{\xi}\right) \\
P_{(p, q)}^{h, T} & \text { s.t. } \quad\left\{\begin{array}{l}
g_{\xi}^{h}\left(z_{\xi}, z_{\xi^{-}} ; p, q\right) \leq 0, \quad \forall \xi \in D^{T}, \\
z_{\xi}=\left(x_{\xi}, \theta_{\xi}, \varphi_{\xi}\right) \geq 0, \quad \forall \xi \in D^{T}, z_{\xi_{0}^{-}}=0 .
\end{array}\right.
\end{aligned}
$$

Note that, there exists a solution for $P_{(p, q)}^{h, T}$ if and only if there is a solution for,

$$
\begin{aligned}
\max & \sum_{\xi \in D^{T}} v_{\xi}^{h}\left(z_{\xi}\right) \\
\tilde{P}_{(p, q)}^{h, T} & \text { s.t. } \begin{cases}g_{\xi}^{h}\left(z_{\xi}, z_{\xi} ; p, q\right) & \leq 0, \quad \forall \xi \in D^{T}, \\
z_{\xi}=\left(x_{\xi}, \theta_{\xi}, \varphi_{\xi}\right) & \geq 0, \quad \forall \xi \in D^{T}, z_{\xi_{0}^{-}}=0, \\
\theta_{\xi, j} & =0, \quad \forall(\xi, j) \in D^{T-1} \times J \text { such that } q_{\xi, j}=0 .\end{cases}
\end{aligned}
$$

Indeed, it follows from the existence of an optimal plan for the consumer problem, giving finite utility, that if $q_{\xi, j}=0$, for some $(\xi, j) \in D \times J$, then $D_{\mu, j}(p, q)=0$ for each $\mu \in \xi^{+}$. Thus, long positions on assets with zero prices do not induce any gains. On the other hand, by Assumption B, commodity prices need to be strictly positive, because we have a finite optimum of individual problem. Also, for any pair $(\xi, j) \in D \times J$, $p_{\xi} C_{\xi, j}-q_{\xi, j}>0$, because otherwise individuals may increase their utilities by increasing their loans (detailed arguments, for the case of short-lived assets, are in Araujo, Páscoa and Torres-Martínez (2002, Proposition $1)$ ). Thus, the set of admissible strategies in $\tilde{P}_{(p, q)}^{h, T}$ is compact and, therefore, by the continuity of the utility function we conclude that there is a solution for $\tilde{P}_{(p, q)}^{h, T}$.

Therefore, for any $T \in \mathbb{N}$, the problem $P_{(p, q)}^{h, T}$ has a solution, $\left(z_{\xi}^{h, T}\right)_{\xi \in D^{T}}=\left(x_{\xi}^{h, T}, \theta_{\xi}^{h, T}, \varphi_{\xi}^{h, T}\right)_{\xi \in D}$. It is immediate that $\sum_{\xi \in D^{T}} v_{\xi}^{h}\left(x_{\xi}^{h, T}+\sum_{j \in J} C_{\xi, j} \varphi_{\xi, j}^{h, T}\right) \leq U^{h}\left(\left(x_{\xi}^{h}+\sum_{j \in J} C_{\xi, j} \varphi_{\xi, j}^{h}\right)_{\xi \in D}\right)$. Thus, there are nonnegative multipliers $\left(\gamma_{\xi}^{h, T}\right)_{\xi \in D^{T}}$ such that, for each nonnegative plan $\left(z_{\xi}\right)_{\xi \in D^{T}} \in \mathbb{Z}^{D^{T}}$, the following saddle point property is satisfied (see Rockafellar (1997), Theorem 28.3),

$$
\sum_{\xi \in D^{T}} \mathcal{L}_{\xi}^{h}\left(z_{\xi}, z_{\xi^{-}}, \gamma_{\xi}^{h, T} ; p, q\right) \leq U^{h}\left(\left(x_{\xi}^{h}+\sum_{j \in J} C_{\xi, j} \varphi_{\xi, j}^{h}\right)_{\xi \in D}\right)
$$

with $\gamma_{\xi}^{h, T} g_{\xi}^{h}\left(z_{\xi}^{h, T}, z_{\xi^{-}}^{h, T} ; p, q\right)=0$

Claim A1. For each $\xi \in D$, the sequence $\left(\gamma_{\xi}^{h, T}\right)_{T \geq t(\xi)}$ is bounded.

Proof. Given $\tilde{D} \subset D$, consider the function $\chi_{\tilde{D}}: D \rightarrow\{0,1\}$ defined by $\chi_{\tilde{D}}(\xi)=1$ if and only if $\xi \in \tilde{D}$. Given $t \leq T$ and evaluating inequality (A.2) in $z=\left(z_{\mu}\right)_{\mu \in D^{T}}$, where $z_{\mu}=\left(W_{\mu}^{h}, 0,0\right) \chi_{D^{t-1}}(\mu)$, we obtain $\sum_{\mu \in D_{t}} \gamma_{\mu}^{h, T} p_{\mu} W_{\mu}^{h} \leq U^{h}\left(\widehat{x}^{h}\right)$. Also, Assumptions A and B imply that, for any $\mu \in D$, both $\min _{l \in L} W_{\mu, l}^{h}$ and $\left\|p_{\mu}\right\|_{\Sigma}$ are strictly positive. Thus, the result follows. 
Claim A2. For each $0<t \leq T$,

$$
0 \leq-\sum_{\xi \in D_{t}} \gamma_{\xi}^{h, T} \nabla_{2} g_{\xi}^{h}(p, q) z_{\xi}^{h} \leq \sum_{\xi \in D \backslash D^{t-1}} v_{\xi}^{h}\left(z_{\xi}^{h}\right)
$$

Proof. Given $t \leq T$, if we evaluate (A.2) in $z=\left(z_{\xi}\right)_{\xi \in D^{T}}$, with $z_{\xi}=z_{\xi}^{h} \chi_{D^{t-1}}(\xi)$, by budget feasibility of allocation $\left(z_{\xi}^{h}\right)_{\xi \in D}$, we have

$$
-\sum_{\xi \in D_{t}} \gamma_{\xi}^{h, T} \nabla_{2} g_{\xi}^{h}(p, q) \cdot z_{\xi}^{h}+\sum_{\xi \in D^{T} \backslash D^{t-1}} \gamma_{\xi}^{h, T} p_{\xi} w_{\xi}^{h} \leq \sum_{\xi \in D \backslash D^{t-1}} v_{\xi}^{h}\left(z_{\xi}^{h}\right)
$$

which implies,

$$
-\sum_{\xi \in D_{t}} \gamma_{\xi}^{h, T} \nabla_{2} g_{\xi}^{h}(p, q) \cdot z_{\xi^{-}}^{h} \leq \sum_{\xi \in D \backslash D^{t-1}} v_{\xi}^{h}\left(z_{\xi}^{h}\right)
$$

This concludes the proof, as the left hand side term in the inequality above is non-negative.

Claim A3. For each $\xi \in D^{T} \backslash D_{T}$ and for any plan $y \geq 0$, we have

$$
v_{\xi}^{h}(y)-v_{\xi}^{h}\left(z_{\xi}^{h}\right) \leq\left(\gamma_{\xi}^{h, T} \nabla_{1} g_{\xi}^{h}(p, q)+\sum_{\mu \in \xi^{+}} \gamma_{\mu}^{h, T} \nabla_{2} g_{\mu}^{h}(p, q)\right)\left(y-z_{\xi}^{h}\right)+\sum_{\eta \in D \backslash D^{T}} v_{\eta}^{h}\left(z_{\eta}^{h}\right) .
$$

Proof. It follows from (A.2) that, given $\xi \in D^{T} \backslash D_{T}$, for each $y \geq 0$, we can choose a plan $z=\left(z_{\mu}\right)_{\mu \in D^{T}}$ with $z_{\mu}=z_{\mu}^{h}\left(1-\chi_{\{\xi\}}(\mu)\right)+y \chi_{\{\xi\}}(\mu)$, in order to guarantee that,

$$
v_{\xi}^{h}(y)-\gamma_{\xi}^{h, T} g_{\xi}^{h}\left(y, z_{\xi^{-}}^{h} ; p, q\right)-\sum_{\mu \in \xi^{+}} \gamma_{\mu}^{h, T} g_{\mu}^{h}\left(z_{\mu}^{h}, y ; p, q\right) \leq v_{\xi}^{h}\left(z_{\xi}^{h}\right)+\sum_{\eta \in D \backslash D^{T}} v_{\eta}^{h}\left(z_{\eta}^{h}\right) .
$$

Now, as the function $g_{\xi}^{h}(\cdot ; p, q)$ is affine and the plan $\left(z_{\xi}^{h}\right)_{\xi \in D} \in B^{h}(p, q)$, we have that,

$$
g_{\xi}^{h}\left(y, z_{\xi^{-}}^{h} ; p, q\right)=\nabla_{1} g_{\xi}^{h}(p, q) y-p_{\xi} w_{\xi}^{h}+\nabla_{2} g_{\xi}^{h}(p, q) z_{\xi^{-}}^{h} \leq \nabla_{1} g_{\xi}^{h}(p, q) y-\nabla_{1} g_{\xi}^{h}(p, q) z_{\xi}^{h},
$$

and, for each node $\mu \in \xi^{+}$,

$$
g_{\mu}^{h}\left(z_{\mu}^{h}, y ; p, q\right)=\nabla_{1} g_{\mu}^{h}(p, q) z_{\mu}^{h}-p_{\mu} w_{\mu}^{h}+\nabla_{2} g_{\mu}^{h}(p, q) y \leq-\nabla_{2} g_{\mu}^{h}(p, q) z_{\xi}^{h}+\nabla_{2} g_{\mu}^{h}(p, q) y .
$$

Substituting the right hand side of inequalities above in equation (A.5) we conclude the proof.

As the event-tree is countable, Tychonoff Theorem and Claim A1 assure the existence of a common subsequence $\left(T_{k}\right)_{k \in \mathbb{N}} \subset \mathbb{N}$ and non-negative multipliers $\left(\gamma_{\xi}^{h}\right)_{\xi \in D}$ such that, for each $\xi \in D, \gamma_{\xi}^{h, T_{k}} \rightarrow_{k \rightarrow+\infty} \gamma_{\xi}^{h}$, and

$$
\begin{aligned}
\gamma_{\xi}^{h} g_{\xi}^{h}\left(p, q, z_{\xi}^{h}, z_{\xi^{-}}^{h}\right) & =0 ; \\
\lim _{t \rightarrow+\infty} \sum_{\xi \in D_{t}} \gamma_{\xi}^{h} \nabla_{2} g_{\xi}^{h}(p, q) z_{\xi^{-}}^{h} & =0,
\end{aligned}
$$

where (A.6) follows from the strictly monotonicity of $u_{\xi}^{h}$, and equation (A.7) is a consequence of Claim A2 (taking first, the limit as $T$ goes to infinity in (A.3) and, afterwards, the limit in $t$ ). Moreover, taking the limit as $T$ goes to infinity in (A.4) we obtain that,

$$
v_{\xi}^{h}(y)-v_{\xi}^{h}\left(z_{\xi}^{h}\right) \leq\left(\gamma_{\xi}^{h} \nabla_{1} g_{\xi}^{h}(p, q)+\sum_{\mu \in \xi^{+}} \gamma_{\mu}^{h} \nabla_{2} g_{\mu}^{h}(p, q)\right)\left(y-z_{\xi}^{h}\right), \quad \forall y \geq 0 .
$$

Therefore, $\gamma_{\xi}^{h} \nabla_{1} g_{\xi}^{h}(p, q)+\sum_{\mu \in \xi^{+}} \gamma_{\mu}^{h} \nabla_{2} g_{\mu}^{h}(p, q) \in \partial^{+} v_{\xi}^{h}\left(z_{\xi}^{h}\right)$, where

$$
\partial^{+} v_{\xi}^{h}(z):=\left\{v_{\xi}^{\prime} \in \mathbb{Z}: v_{\xi}^{h}(y)-v_{\xi}^{h}(z) \leq v_{\xi}^{\prime} \cdot(y-z), \quad \forall y \geq 0\right\} .
$$


That is, $\partial^{+} v_{\xi}^{h}(\cdot)$ is the super-differential of the function $v_{\xi}^{h}(\cdot)+\delta\left(\cdot, \mathbb{R}_{+}^{L}\right)$, where $\delta\left(z, \mathbb{R}_{+}^{L}\right)=0$, when $z \geq 0$ and $\delta\left(z, \mathbb{R}_{+}^{L}\right)=-\infty$, in other case. Notice that, for each $z \geq 0, \kappa \in \partial \delta(z) \Leftrightarrow 0 \leq k(y-z), \forall y \geq 0$. Thus, by Theorem 23.8 in Rockafellar (1997), for all $z \geq 0$, if $v_{\xi}^{\prime} \in \partial^{+} v_{\xi}^{h}(z)$ then there exists $\tilde{v}_{\xi}^{\prime} \in \partial v_{\xi}^{h}(z)$ such that both $v_{\xi}^{\prime} \geq \tilde{v}_{\xi}^{\prime}$ and $\left(v_{\xi}^{\prime}-\tilde{v}_{\xi}^{\prime}\right) \cdot z=0$. Thus, it follows from (A.8) that there exists, for each $\xi \in D$, a super-gradient $\tilde{v}_{\xi}^{\prime} \in \partial v_{\xi}^{h}\left(z_{\xi}^{h}\right)$ such that,

$$
\begin{aligned}
\gamma_{\xi}^{h} \nabla_{1} g_{\xi}^{h}(p, q)+\sum_{\mu \in \xi^{+}} \gamma_{\mu}^{h} \nabla_{2} g_{\mu}^{h}(p, q) & \geq \tilde{v}_{\xi}^{\prime}, \\
\left(\gamma_{\xi}^{h} \nabla_{1} g_{\xi}^{h}(p, q)+\sum_{\mu \in \xi^{+}} \gamma_{\mu}^{h} \nabla_{2} g_{\mu}^{h}(p, q)\right) z_{\xi}^{h} & =\tilde{v}_{\xi}^{\prime} z_{\xi}^{h} .
\end{aligned}
$$

By definition, $\left(\tilde{v}_{\xi}^{\prime}-\gamma_{\xi}^{h} \nabla_{1} g_{\xi}^{h}(p, q),-\gamma_{\xi}^{h} \nabla_{2} g_{\xi}^{h}(p, q)\right) \in \partial \mathcal{L}_{\xi}^{h}\left(z_{\xi}^{h}, z_{\xi^{-}}^{h}, \gamma_{\xi}^{h} ; p, q\right)$. Therefore, there exists, for each $\xi \in D$, a vector $\left(\mathcal{L}_{\xi, 1}^{\prime}, \mathcal{L}_{\xi, 2}^{\prime}\right) \in \mathcal{L}_{\xi}^{h}\left(z_{\xi}^{h}, z_{\xi}^{h}, \gamma_{\xi}^{h} ; p, q\right)$ satisfying Euler conditions. Furthermore, the transversality condition is a direct consequence of equation (A.7) jointly with Euler conditions. Indeed,

$$
\sum_{\xi \in D_{t-1}} \mathcal{L}_{\xi, 1}^{\prime} z_{\xi}^{h}=-\sum_{\xi \in D_{t}} \mathcal{L}_{\xi, 2}^{\prime} z_{\xi^{-}}^{h}=\sum_{\xi \in D_{t}} \gamma_{\xi}^{h} \nabla_{2} g_{\xi}^{h}(p, q) z_{\xi^{-}}^{h} \rightarrow_{t \rightarrow+\infty} 0
$$

On the other hand, it follows from Euler equations, using the sign property of the Lagrangian, that $\tilde{v}_{\xi}^{\prime}-\gamma_{\xi}^{h} \nabla_{1} g_{\xi}^{h}(p, q) \leq 0$. As utility functions $u_{\xi}^{h}$ are strictly increasing in the first argument, we know that $\tilde{v}_{\xi}^{\prime}$ has a strictly positive first coordinate. Thus, we have that $\gamma_{\xi}^{h} p_{\xi, 1}>0$, which implies that the multipliers $\gamma_{\xi}^{h}$ are strictly positive, for each $\xi \in D$.

Therefore, there is a plan of Kuhn-Tucker multipliers associated with $\left(z_{\xi}^{h}\right)_{\xi \in D}$.

(ii) It follows from (EE) that, for each $T \geq 0$,

$$
\sum_{\xi \in D^{T}} \mathcal{L}_{\xi}^{h}\left(z_{\xi}, z_{\xi^{-}}, \gamma_{\xi}^{h} ; p, q\right)-\sum_{\xi \in D^{T}} \mathcal{L}_{\xi}^{h}\left(z_{\xi}^{h}, z_{\xi^{-}}^{h}, \gamma_{\xi}^{h} ; p, q\right) \leq \sum_{\xi \in D_{T}} \mathcal{L}_{\xi, 1}^{\prime}\left(z_{\xi}-z_{\xi}^{h}\right) .
$$

Since, at any node $\xi \in D$ we have that $\gamma_{\xi}^{h} g_{\xi}\left(z_{\xi}^{h}, z_{\xi^{-}}^{h} ; p, q\right)=0$, each $\left(z_{\xi}\right)_{\xi \in D} \in B^{h}(p, q)$ must satisfy

$$
\sum_{\xi \in D^{T}} u_{\xi}^{h}\left(\widehat{x}_{\xi}\right)-\sum_{\xi \in D^{T}} u_{\xi}^{h}\left(\widehat{x}_{\xi}^{h}\right) \leq \sum_{\xi \in D_{T}} \mathcal{L}_{\xi, 1}^{\prime}\left(z_{\xi}-z_{\xi}^{h}\right)
$$

Using the condition (TC) we have that $U^{h}(\widehat{x})-U^{h}\left(\widehat{x}^{h}\right) \leq \lim \sup _{T \rightarrow+\infty} \sum_{\xi \in D_{T}} \mathcal{L}_{\xi, 1}^{\prime} z_{\xi}$.

Also, Euler conditions imply that $\sum_{\xi \in D_{T}} \mathcal{L}_{\xi, 1}^{\prime} z_{\xi} \leq-\sum_{\mu \in D_{T+1}} \mathcal{L}_{\mu, 2}^{\prime} z_{\mu^{-}} \leq 0$, where the last inequality follows from the sign property $\mathcal{L}_{\mu, 2}^{\prime} \geq 0$, satisfied at each node of the event-tree. Thus, $U^{h}(\widehat{x}) \leq U^{h}\left(\widehat{x}^{h}\right)$, which guarantees that the allocation $\left(z_{\xi}^{h}\right)_{\xi \in D}$ solves $P_{(p, q)}^{h}$. Moreover, when $x_{\xi}^{h}+\sum_{j \in J} C_{\xi, j} \varphi_{\xi, j}^{h} \leq \mathbb{W}_{\xi}$, for each $\xi \in D$, Assumption $\mathrm{B}$ assures that the optimum value is finite.

(iii) As we pointed out in inequality (A.10), the existence of Kuhn-Tucker multipliers $\left(\gamma_{\xi}^{h}\right)_{\xi \in D}$ implies that, for any $T>0, \sum_{\xi \in D^{T}} \mathcal{L}_{\xi}^{h}\left(0,0, \gamma_{\xi}^{h} ; p, q\right)-\sum_{\xi \in D^{T}} \mathcal{L}_{\xi}^{h}\left(z_{\xi}^{h}, z_{\xi^{-}}^{h}, \gamma_{\xi}^{h} ; p, q\right) \leq-\sum_{\xi \in D_{T}} \mathcal{L}_{\xi, 1}^{\prime} z_{\xi}^{h}$, and, therefore, $\sum_{\xi \in D^{T}} \gamma_{\xi}^{h} p_{\xi} w_{\xi}^{h} \leq U^{h}\left(\widehat{x}^{h}\right)-\sum_{\xi \in D_{T}} \mathcal{L}_{\xi, 1}^{\prime} z_{\xi}^{h}$. Using the transversality condition (TC), we conclude that $\sum_{\xi \in D} \gamma_{\xi}^{h} p_{\xi} w_{\xi}^{h}<+\infty$.

Proof of Claims after Proposition 1. Budget feasibility and Assumption B implies that

$$
{ }_{T \rightarrow+\infty} \sum_{\mu \in D_{T}} \gamma_{\mu}^{h} \nabla_{2} g_{\mu}^{h}(p, q) z_{\mu^{-}}^{h}=\lim _{T \rightarrow+\infty} \sum_{\mu \in D_{T}} \gamma_{\mu}^{h} \nabla_{1} g_{\mu}^{h}(p, q) z_{\mu}^{h}-\lim _{T \rightarrow+\infty} \sum_{\mu \in D_{T}} \gamma_{\mu}^{h} p_{\mu} w_{\mu}^{h}
$$


Therefore, as deflated endowments are summable, using Euler conditions we assure that our transversality condition is equivalent to $\lim _{T \rightarrow+\infty} \sum_{\mu \in D_{T}} \gamma_{\mu}^{h} \nabla_{1} g_{\mu}^{h}(p, q) z_{\mu}^{h}=0$.

Proof of Corollary 1. Since under prices $(p, q) \in \mathbb{P}$ agent $h$ 's problem has a finite optimum, denote by $z^{h}:=\left(z_{\xi}^{h}\right)_{\xi \in D}$ the optimal plan of agent $h$ at prices $(p, q)$. It follows from Proposition 1-(i) that there is a plan of Kuhn-Tucker multipliers associated with $z^{h}$.

Thus, there are $\left(\mathcal{L}_{\xi, 1}^{\prime}, \mathcal{L}_{\xi, 2}^{\prime}\right)_{\xi \in D} \in \prod_{\xi \in D} \partial \mathcal{L}_{\xi}^{h}\left(z_{\xi}^{h}, z_{\xi^{-}}^{h}, \gamma_{\xi}^{h} ; p, q\right)$ such that, for any $\xi \in D, \mathcal{L}_{\xi, 1}^{\prime}+\sum_{\mu \in \xi^{+}} \mathcal{L}_{\mu, 2}^{\prime} \leq$ 0 . Using the characterization of $\left(\mathcal{L}_{\xi, 1}^{\prime}, \mathcal{L}_{\xi, 2}^{\prime}\right)_{\xi \in D}$ at the beginning of this Appendix and the fact that $v_{\xi}^{\prime} \in$ $\partial v_{\xi}^{h}\left(z_{\xi}^{h}\right)$ if and only if there is $\alpha_{\xi} \in \partial u_{\xi}^{h}\left(\widehat{x}_{\xi}^{h}\right)$ such that $v_{\xi}^{\prime}=\left(\alpha_{\xi}, 0,\left(\alpha_{\xi} C_{\xi, j}\right)_{j \in J}\right)$, we obtain inequalities (5)-(7), as the super gradients of $u_{\xi}^{h}$ are vectors with strictly positive entries.

On the other hand, fix $(\xi, j) \in D \times J$. Using the notation introduced after Definition 3, inequalities (5)-(7) imply that, $\eta_{\varphi}(\Gamma, \xi, j)=\sum_{l \in L} \eta_{x}(\Gamma, \xi, l) C_{\xi, j, l}-\eta_{\theta}(\Gamma, \xi, j)$. Therefore, if for each $l \in L$ for which $C_{\xi, j, l} \neq 0$ inequality (5) holds as equality, then $\eta_{\theta}(\Gamma, \xi, j)=\eta_{\varphi}(\Gamma, \xi, j)=0$, which implies that inequalities (6) and (7) holds as equalities.

\section{Appendix B. Transversality conditions of Example 1.}

Transversality condition in long positions,

$$
\begin{aligned}
\sum_{\eta \in D_{T}} \gamma_{\eta}^{1} q_{\eta} \theta_{\eta}^{1} & =\kappa e \frac{1}{2(1-\kappa)} \sum_{\left\{\eta \in D_{T}: \eta=\left(\eta^{-}\right)^{u}\right\}} \rho^{1}(\eta)=\kappa e \frac{1}{2(1-\kappa)} \frac{1}{2^{T}} \longrightarrow 0 \\
\sum_{\eta \in D_{T}} \gamma_{\eta}^{2} q_{\eta} \theta_{\eta}^{2} & =\kappa e \frac{1}{2(1-\kappa)} \sum_{\left\{\eta \in D_{T}: \eta=\left(\eta^{-}\right)^{d}\right\}} \rho^{2}(\eta)=\kappa e \frac{1}{2(1-\kappa)} \frac{1}{2^{T}} \longrightarrow 0 .
\end{aligned}
$$

Tranversality condition in consumption,

$$
\begin{aligned}
\sum_{\eta \in D_{T}} \gamma_{\eta}^{h} p_{\eta, x} \widehat{x}_{\eta, 1}^{h} & =\beta^{T} \sum_{\eta \in D_{T}} \rho^{h}(\eta)\left(1+d_{\eta}^{h^{\prime}}\right)=\beta^{T}+\frac{\kappa e}{2(1-\kappa)} \sum_{\left\{\eta \in D_{T}: d_{\eta}^{h^{\prime}} \neq 0\right\}} \rho^{h}(\eta) \\
& \leq \beta^{T}+\frac{\kappa e}{2(1-\kappa)} \frac{1}{2^{T-1}}\left(1-\frac{1}{2^{T}}\right) \sum_{\eta \in D_{T-2}} \rho^{h}(\eta) \longrightarrow 0 ; \\
\sum_{\eta \in D_{T}} \gamma_{\eta}^{h} p_{\eta, y} \widehat{x}_{\eta, 2}^{h} & =\delta^{T} \frac{1}{2(1-\kappa)} \longrightarrow 0 .
\end{aligned}
$$

\section{Appendix C. Proof of Theorem 1.}

An equilibrium for the infinite horizon economy will be found as a limit of equilibria of truncated economies, when the time horizon goes to infinity.

Equilibria in truncated economies. Define, for each $T \in \mathbb{N}$, a truncated economy, $\mathcal{E}^{T}$, in which agents are restricted to consume and trade assets in the event-tree $D^{T}$. Thus, given prices $(p, q)$ in $\mathbb{P}^{T}:=\{(p, q)=$ $\left.\left(p_{\xi}, q_{\xi}\right)_{\xi \in D^{T}} \in\left(\mathbb{R}_{+}^{L} \times \mathbb{R}_{+}^{J}\right)^{D^{T}}:\left\|p_{\xi}\right\|_{\Sigma}+\left\|q_{\xi}\right\|_{\Sigma}=1, \quad \forall \xi \in D^{T}\right\}$, each agent $h \in H$ has the objective to choose, at each $\xi \in D^{T}$, a vector $z_{\xi}^{h, T}=\left(x_{\xi}^{h, T}, \theta_{\xi}^{h, T}, \varphi_{\xi}^{h, T}\right) \in \mathbb{Z}$ in order to solve the (truncated) individual problem $P_{(p, q)}^{h, T}$ defined at the beginning of proof of Proposition 1. Now, let $B^{h, T}(p, q)$ be the truncated budget set of agent $h$ in $\mathcal{E}^{T}$. That is, the set of plans $\left(z_{\xi}\right)_{\xi \in D^{T}}$ that satisfy the restrictions of problem $P_{(p, q)}^{h, T}$.

An equilibrium for $\mathcal{E}^{T}$ is given by prices $\left(p^{T}, q^{T}\right) \in \mathbb{P}^{T}$ jointly with delivery rates $\lambda_{\xi}^{T}=\left(\lambda_{\xi, j}^{T}\right)$, for each $\xi \in D^{T} \backslash\left\{\xi_{0}\right\}$, and individual plans $z_{\xi}^{h, T}=\left(x_{\xi}^{h, T}, \theta_{\xi}^{h, T}, \varphi_{\xi}^{h, T}\right)_{\xi \in D^{T}}$ such that: (1) $z^{h, T}$ is an optimal solution for $P_{\left(p^{T}, q^{T}\right)}^{h, T}$; (2) physical and financial market clear node by node in $D^{T}$, in the sense of Definition 1 ; and 
(3) for each $\xi \in D^{T} \backslash\left\{\xi_{0}\right\}$, delivery rates $\lambda_{\xi, j}^{T}$ satisfy condition B of Definition 1 .

Note that, market feasible allocations, that is, the non-negative allocations $\left(x_{\xi}^{h}, \theta_{\xi}^{h}, \varphi_{\xi}^{h}\right)_{(h, \xi) \in H \times D^{T}}$ that satisfy market clearing conditions $\mathrm{C}$ and D of Definition 1, are bounded in $D^{T} .{ }^{10}$ Therefore, departing from $\mathcal{E}^{T}$ we can define a compact economy $\mathcal{E}^{T}\left(K^{T}\right)$ by restricting the space of plans of each $h \in H$ to the convex and compact set $K^{T}:=\left\{z=(x, \theta, \varphi) \in \mathbb{R}_{+}^{L \times D^{T}} \times \mathbb{R}_{+}^{J \times D^{T}} \times \mathbb{R}_{+}^{J \times D^{T}}:\|z\|_{\Sigma} \leq 2 \Upsilon^{T}\right\}$, which has in its interior the vector $\Upsilon^{T}$ that is defined as an upper bound for the feasible allocations in $D^{T}$.

An equilibrium for $\mathcal{E}\left(K^{T}\right)$ is given by prices $\left(p^{T}, q^{T}\right) \in \mathbb{P}^{T}$, delivery rates $\lambda_{\xi}^{T}=\left(\lambda_{\xi, j}^{T}\right)$, for each node $\xi \in D^{T} \backslash\left\{\xi_{0}\right\}$, and allocations $\left(z_{\xi}^{h, T}\right)_{\xi \in D^{T}}=\left(x_{\xi}^{h, T}, \theta_{\xi}^{h, T}, \varphi_{\xi}^{h, T}\right)_{\xi \in D^{T}}$, compatible with conditions B, C and D of Definition 1, such that, for each agent $h$, the plan $\left(z_{\xi}^{h, T}\right)_{\xi \in D^{T}}$ solves,

$\left(P_{\left(p^{T}, q^{T}\right)}^{h, T}\left(K^{T}\right)\right)$

$$
\begin{array}{ll}
\max & \sum_{\xi \in D^{T}} v_{\xi}^{h}\left(z_{\xi}\right) \\
\text { s.t. } & \left(z_{\xi}\right)_{\xi \in D^{T}} \in B^{h, T}\left(p^{T}, q^{T}\right) \cap K^{T} .
\end{array}
$$

If we assure the existence of equilibrium for $\mathcal{E}^{T}\left(K^{T}\right)$, the economy $\mathcal{E}^{T}$ has also an equilibrium, given that optimal allocation of $\mathcal{E}^{T}\left(K^{T}\right)$ will be, by construction, interior points of set $K^{T}$, budget sets are convex and utility functions are concave under Assumption B.

Generalized Games. To prove the existence of equilibrium in $\mathcal{E}^{T}\left(K^{T}\right)$ we introduce a game $\mathcal{G}^{T}$, where each $h \in H$ takes prices $(p, q) \in \mathbb{P}^{T}$ as given and solves the compact truncated problem above. Moreover, associated with each $(\xi, j) \in\left(D^{T} \backslash\left\{\xi_{0}\right\}\right) \times J$, there is one fictitious player that, given prices $(p, q) \in \mathbb{P}^{T}$, will be choose a real number $\lambda_{\xi, j} \in[0,1]$ in order to solve the problem,

$$
\min _{\lambda \in[0,1]}\left[\lambda\left(p_{\xi} A(\xi, j)+q_{\xi, j}\right)+(1-\lambda) p_{\xi} \widehat{C}_{\xi, j}\right] .
$$

Finally, associated to each node in $D^{T}$ there is an auctioneer who, given plans $\left(z_{\xi}^{h}\right)_{(h, \xi) \in H \times D^{T}} \in \prod_{h \in H} K^{T}$ and delivery rates $\lambda_{\xi, j} \in[0,1]$ has the objective to find prices $\left(p_{\xi}, q_{\xi}\right) \in \Delta_{+}^{L+J-1}$ in order to maximize the function,

$$
\begin{aligned}
& p_{\xi} \sum_{h \in H}\left(x_{\xi}^{h}+\sum_{j \in J} C_{\xi, j} \varphi_{\xi, j}^{h}-w_{\xi}^{h}-Y_{\xi} x_{\xi^{-}}^{h}-Y_{\xi} \sum_{j \in J} C_{\xi^{-}, j} \varphi_{\xi^{-}, j}^{h}\right) \\
&+\sum_{j \in J} q_{\xi, j} \sum_{h \in H}\left(\theta_{\xi, j}^{h}-\varphi_{\xi, j}^{h}-\lambda_{\xi, j}\left(\theta_{\xi^{-}, j}^{h}-\varphi_{\xi^{-}, j}^{h}\right)\right) \\
& \quad-p_{\xi} \sum_{(h, j) \in H \times J}\left(\lambda_{\xi, j} A(\xi, j)+\left(1-\lambda_{\xi, j}\right) \widehat{C}_{\xi, j}\right)\left(\theta_{\xi^{-}, j}^{h}-\varphi_{\xi^{-}, j}^{h}\right),
\end{aligned}
$$

where, $z_{\xi}^{h}=\left(x_{\xi}^{h}, \theta_{\xi}^{h}, \varphi_{\xi}^{h}\right)$ and, for convenience of notations, for each $(h, j) \in H \times J$ we put $\left(x_{\xi_{0}^{-}}^{h}, \theta_{\xi_{0}^{-}, j}^{h}, \varphi_{\xi_{0}^{-}, j}^{h}\right)=$ $\left(0, e_{j}^{h}, 0\right)$ and $\left(\widehat{C}_{\xi_{0}, j}, Y_{\xi_{0}}\right)=(0,0)$, for all $j \in J$

A vector $\left[\left(p^{T}, q^{T}\right) ;\left(z_{\xi}^{h, T}\right)_{h \in H} ; \lambda_{\xi}^{T}\right]_{\xi \in D^{T}}$ that solves simultaneously the problems above is called a (CournotNash) equilibrium of $\mathcal{G}^{T}$.

\footnotetext{
${ }^{10}$ Indeed, autonomous consumption allocations, $\left(x_{\xi}^{h}\right)_{(h, \xi) \in H \times D^{T}}$ are bounded by above, node by node, by the aggregated physical endowments. The short-sales $\left(\varphi_{\xi, j}^{h}\right)_{(h, \xi) \in H \times D^{T}}$ are bounded, at each $\xi \in D^{T}$, by $\sum_{l \in L} \mathbb{W}_{\xi, l}$ divided by the positive number $\left\|C_{\xi, j}\right\|_{\Sigma}$. Thus, long positions $\left(\theta_{\xi, j}^{h}\right)_{(h, \xi) \in H \times D^{T}}$ are also bounded, because are less than or equal to the aggregate short sales plus the initial positive net supply.
} 
Lemma C1. For each $T \in \mathbb{N}$ there is an equilibrium for $\mathcal{G}^{T}$.

Proof. The objective function of each participant in the game is continuous and quasi-concave in the own strategy. For fictitious players and auctioneers, the correspondences of admissible strategies are continuous, with non-empty, convex and compact values. Also, the budget restriction correspondence of each agent, $(p, q) \rightarrow B^{h, T}(p, q) \cap K^{T}$, has non-empty, convex and compact values. Therefore, in order to find an equilibrium of the generalized game (as a fixed point of the set function given by the product of optimal strategies correspondences), it is sufficient to prove that budget set correspondences are continuous.

The upper hemi-continuity follows from compact values and closed graph properties, that are a direct consequence of continuity of functions $g_{\xi}^{h}$. Thus, the main difficulty resides in showing the lower hemi-continuity property. Now, as for each price $(p, q) \in \mathbb{P}^{T}$ the set $B^{h, T}(p, q) \cap K^{T}$ is convex and compact, it is sufficient to assure that the (relative) interior correspondence $(p, q) \rightarrow \operatorname{int}\left(B^{h, T}(p, q)\right) \cap K^{T}$ has non-empty values. But this last property follows from Assumption A. In fact, cumulated endowments are such that $W_{\xi}^{h} \gg 0$, for each $h \in H$, and, therefore, given any plan of prices $(p, q) \in \mathbb{P}^{T}$, the plan $\left(\tilde{x}_{\xi}^{h} ; \tilde{\theta}_{\xi}^{h} ; \tilde{\varphi}_{\xi}^{h}\right)_{\xi \in D^{T}}:=\left(\frac{W_{\xi}^{h}}{2^{t(\xi)+1}}-\sum_{j \in J} C_{\xi, j} \epsilon_{\xi}^{h} ; 0 ; \epsilon_{\xi}(1,1, \ldots, 1)\right)_{\xi \in D^{T}}$, where for each $\xi \in D^{T}$, $\epsilon_{\xi}^{h}=\min _{(l, \mu) \in L \times \xi^{+}}\left\{\frac{W_{\xi, l}^{h}}{2^{t(\xi)+2}\left(1+\sum_{j \in J}\left(C_{\xi, j}\right) l\right.} ; \frac{W_{\mu, l}^{h}}{2^{t(\xi)+2}\left(1+\sum_{j \in J} Y_{\mu}(l, \cdot) C_{\xi, j}\right)}\right\}$, is budget feasible and belongs to the relative interior of the set $B^{h, T}(p, q) \cap K$.

Lemma C2. For each $T \in \mathbb{N}$ there is an equilibrium for $\mathcal{E}^{T}\left(K^{T}\right)$.

Proof. We know that there exists an equilibrium for $\mathcal{G}^{T}$, namely $\left[\left(p^{T}, q^{T}\right) ;\left(z_{\xi}^{h, T}\right)_{h \in H} ; \lambda_{\xi}^{T}\right]_{\xi \in D^{T}}$. By definition, the payment rates $\lambda_{\xi, j}^{h, T}$ satisfy condition B of Definition 1 and each agent $h \in H$ solves problem $P^{h, T}\left(K^{T}\right)$ by choosing the plan $\left(z_{\xi}^{h, T}\right)_{\xi \in D^{T}}$. Thus, it is sufficient to verify, for each node $\xi \in D^{T}$, the validity of conditions $\mathrm{C}$ and $\mathrm{D}$ of Definition 1.

Now, it follows from players' objective functions that, for each node $\xi>\xi_{0}$, the effective payments satisfy, $D_{\xi, j}\left(p^{T}, q^{T}\right)=\lambda_{\xi, j}^{T}\left(p_{\xi}^{T} A(\xi, j)+q_{\xi, j}^{T}\right)+\left(1-\lambda_{\xi, j}^{T}\right) p_{\xi}^{T} \widehat{C}_{\xi, j}$. Therefore, as budget feasibility implies that $\sum_{h \in H} g_{\xi}^{h}\left(z_{\xi}^{h, T}, z_{\xi-}^{h, T}, p^{T}, q^{T}\right) \leq 0$, the optimal value of auctioneers objective functions is less than or equal to zero. This implies that conditions $\mathrm{C}$ and D of Definition 1 are satisfied as inequalities. That is, there does not exist excess demand in physical and financial markets.

Thus, as the individual demands for commodities or assets are bounded by the aggregate supply of resources, the optimal bundles that were chosen by the agents are interior points of $K^{T}$. Therefore, monotonicity of utility function implies that, for each $\xi \in D^{T}, \sum_{h \in H} g_{\xi}^{h}\left(z_{\xi}^{h, T}, z_{\xi}^{h, T}, p^{T}, q^{T}\right)=0$. In other words, Walras' law holds.

The existence of an optimal solution for $P^{h, T}\left(K^{T}\right)$ in the interior of the set $K^{T}$ implies that $p_{\xi}^{T} \gg 0$ and, therefore, condition $\mathrm{C}$ of Definition 1 holds, as a direct consequence of Walras' law, strictly positive commodity prices and the absence of excess demand in physical markets. By analogous arguments, condition D of Definition 1 holds, at a node $\xi \in D^{T}$, for those assets $j \in J$ which have a strictly positive price $q_{\xi, j}^{T}>0$.

Given $\xi \in D^{T}$, denote by $\tilde{J}_{\xi} \subset J$ the set of assets with zero price at $\xi$ and let $\Delta\left(\theta_{\xi}^{T}, \theta_{\xi^{-}}^{T}\right)_{\xi, j}$ be the excess demand of asset $j$ at node $\xi$, associated with long positions $\left(\theta_{\xi}^{T}, \theta_{\xi}^{T}\right)=\left(\theta_{\xi}^{h, T}, \theta_{\xi^{-}}^{h, T}\right)_{h \in H}$ (it follows from previous arguments that $\left.\Delta\left(\theta_{\xi}^{T}, \theta_{\xi^{-}}^{T}\right)_{\xi, j} \leq 0\right)$. If $j \in \tilde{J}_{\xi}$, then optimality of agents' allocations assures that the asset does not deliver any payment at the successor nodes $\mu \in \xi^{+}$(if this nodes are in $D^{T}$ ). Therefore, if we change the portfolio allocation $\left(\theta_{\xi}^{h, T}\right)_{h \in H}$ to $\tilde{\theta}_{\xi}^{h, T}=\theta_{\xi}^{h, T}-\frac{1}{\# H} \Delta\left(\theta_{\xi}^{T}, \theta_{\xi^{-}}^{T}\right)_{\xi, j}$, we assure that, at node 
$\xi$, and for asset $j$, condition D holds. Moreover, the new allocation is budget feasible, optimal, and we do not lose the market clearing condition in physical markets at node $\mu \in \xi^{+}$.

However, the total supply of asset $j$ at nodes $\mu \in \xi^{+}$can change. Therefore, in order to apply the trick above, node by node, asset by asset, to obtain an optimal allocation that satisfies Condition D for each asset, it is sufficient to prove that, after changing portfolios at a node $\xi$, the new excess demand, at nodes $\mu \in \xi^{+}, \Delta\left(\theta_{\mu}^{T}, \tilde{\theta}_{\xi}^{T}\right)_{\mu, j}$ is still less than or equal to zero and that $\Delta\left(\theta_{\mu}^{T}, \tilde{\theta}_{\xi}^{T}\right)_{\mu, j}$ can be negative only for assets in $\tilde{J}_{\mu}$.

Fix $j \in \tilde{J}_{\xi}$. It follows by the definition of $\tilde{\theta}_{\xi}^{h, T}$ that, at any $\mu \in \xi^{+}, \Delta\left(\theta_{\mu}^{T}, \tilde{\theta}_{\xi}^{T}\right)_{\mu, j} \leq \Delta\left(\theta_{\mu}^{T}, \theta_{\xi}^{T}\right)_{\mu, j}$. Now, as at each $\mu \in \xi^{+}, D_{\mu, j}\left(p^{T}, q^{T}\right)=0$ then asset $j$ defaults at nodes $\mu \in \xi^{+}$. Therefore, $\left(\lambda_{\mu, j}^{T}\right)_{\mu \in \xi^{+}}=0$ and $\left(\Delta\left(\theta_{\mu}^{T}, \tilde{\theta}_{\xi}^{T}\right)_{\mu, j}\right)_{\mu \in \xi^{+}}=\left(\Delta\left(\theta_{\mu}^{T}, \theta_{\xi}^{T},\right)_{\mu, j}\right)_{\mu \in \xi^{+}}$, which concludes the proof.

In the previous lemma we found an equilibrium for $\mathcal{E}^{T}\left(K^{T}\right)$. It is not difficult to verify that this equilibrium constitutes also an equilibrium for $\mathcal{E}^{T}$.

Asymptotic equilibria. For each $T \in \mathbb{N}$, fix an equilibrium $\left[\left(p^{T}, q^{T}\right) ;\left(z_{\xi}^{h, T}\right)_{h \in H} ; \lambda_{\xi}^{T}\right]_{\xi \in D^{T}}$ of $\mathcal{E}^{T}$. We know that there exist non-negative multipliers $\left(\gamma_{\xi}^{h, T}\right)_{\xi \in D^{T}}$ such that, $\gamma_{\xi}^{h, T} g_{\xi}^{h}\left(z_{\xi}^{h, T}, z_{\xi^{-}}^{h, T} ; p, q\right)=0$, and the following saddle point property is satisfied, for each nonnegative plan $\left(z_{\xi}\right)_{\xi \in D^{T}}$ (see Rockafellar (1997), Section 28, Theorem 28.3),

$$
\sum_{\xi \in D^{T}} \mathcal{L}_{\xi}^{h}\left(z_{\xi}, z_{\xi^{-}}, \gamma_{\xi}^{h, T} ; p^{T}, q^{T}\right) \leq \sum_{\xi \in D^{T}} v_{\xi}^{h}\left(z_{\xi}^{h, T}\right) .
$$

As $v_{\xi}^{h}\left(z_{\xi}^{h, T}\right) \leq u_{\xi}^{h}\left(\mathbb{W}_{\xi}\right)$, analogously to Claim A1 in Appendix A, for each $\xi \in D$ and for all $T \geq t(\xi)$,

$$
0 \leq \gamma_{\xi}^{h, T}<\frac{U^{h}(\mathbb{W})}{\underline{W}_{\xi}^{h}\left\|p_{\xi}^{T}\right\|_{\Sigma}}
$$

where $\underline{W}_{\xi}^{h}=\min _{l \in L} W_{\xi, l}^{h}>0$.

Lemma C3. For each $\xi \in D$, there is a strictly positive lower bound for $\left(\left\|p_{\xi}^{T}\right\|_{\Sigma}\right)_{T>t(\xi)}$.

Proof. Given $\xi \in D$ and $T>t(\xi)$, optimality of $z^{h, T}$ in $P_{\left(p^{T}, q^{T}\right)}^{h, T}$ implies that $p_{\xi}^{T} C_{\xi, j} \geq q_{\xi, j}^{T}$, for each $j \in J$. Thus, for each $j \in J$, there is $\bar{m}_{\xi, j}>0$ such that, $q_{\xi, j}^{T} \leq \bar{m}_{\xi, j}\left\|p_{\xi}^{T}\right\|_{\Sigma}$. Adding in $j$, we obtain that $\left\|q_{\xi}^{T}\right\|_{\Sigma} \leq\left\|p_{\xi}^{T}\right\|_{\Sigma} \sum_{\mathrm{j} \in J} \bar{m}_{\xi, j}$. Finally, as $\left\|q_{\xi}^{T}\right\|_{\Sigma}=1-\left\|p_{\xi}^{T}\right\|_{\Sigma}$, at each node $\xi \in D$, independently of $T$, $\left\|p_{\xi}^{T}\right\|_{\Sigma} \geq \frac{1}{1+\sum_{\mathrm{\jmath}} \in J} \bar{m}_{\xi, j}>0$.

Therefore, the sequence $\left[\left(p_{\xi}^{T}, q_{\xi}^{T}\right) ;\left(z_{\xi}^{h, T}, \gamma_{\xi}^{h, T}\right)_{h \in H} ; \lambda_{\xi}^{T}\right]_{T>t(\xi)}$ is bounded. Applying Tychonoff Theorem we find, as in the proof of Proposition 1, a subsequence $\left(T_{k}\right)_{k \in \mathbb{N}} \subset \mathbb{N}$ such that, for each $\xi \in D$, $\left[\left(p_{\xi}^{T_{k}}, q_{\xi}^{T_{k}}\right) ;\left(z_{\xi}^{h, T_{k}}, \gamma_{\xi}^{h, T_{k}}\right)_{h \in H} ; \lambda_{\xi}^{T_{k}}\right]_{T_{k}>t(\xi)}$ converges, as $k$ goes to infinity, to an allocation $\left[\left(\bar{p}_{\xi}, \bar{q}_{\xi}\right) ;\left(\bar{z}_{\xi}^{h}, \bar{\gamma}_{\xi}^{h}\right)_{h \in H} ; \bar{\lambda}_{\xi}\right]$.

Moreover, the limit allocations $\left[\left(\bar{z}_{\xi}^{h}\right)_{\xi \in D}\right]_{h \in H}$ are budget feasible at prices $(\bar{p}, \bar{q}) \in \mathbb{P}$, and satisfy market feasibility conditions at each node in the event-tree. Thus, in order to assure that $\left[\left(\bar{p}_{\xi}, \bar{q}_{\xi}\right) ;\left(\bar{z}_{\xi}^{h}, \bar{\gamma}_{\xi}^{h}\right)_{h \in H} ; \bar{\lambda}_{\xi}\right]_{\xi \in D}$ is an equilibrium we just need, by the results of Section 4 , to verify that, for each agent $h \in H,\left(\bar{z}_{\xi}^{h}, \bar{\gamma}_{\xi}^{h}\right)_{\xi \in D}$ satisfies Euler and transversality conditions.

Lemma C4. For each $t>0$ we have that,

$$
0 \leq-\sum_{\xi \in D_{t}} \bar{\gamma}_{\xi}^{h} \nabla_{2} g_{\xi}^{h}(\bar{p}, \bar{q}) \cdot \bar{z}_{\xi}^{h} \leq \sum_{\xi \in D \backslash D^{t-1}} v_{\xi}^{h}\left(\bar{z}_{\xi}^{h}\right)
$$


Moreover, for each $\xi \in D$ and for all plan $y \geq 0$, we have that

$$
v_{\xi}^{h}(y)-v_{\xi}^{h}\left(\bar{z}_{\xi}^{h}\right) \leq\left(\bar{\gamma}_{\xi}^{h} \nabla_{1} g_{\xi}^{h}(\bar{p}, \bar{q})+\sum_{\mu \in \xi^{+}} \bar{\gamma}_{\mu}^{h} \nabla_{2} g_{\mu}^{h}(\bar{p}, \bar{q})\right) \cdot\left(y-\bar{z}_{\xi}^{h}\right) .
$$

Proof. The proof is analogous to those made in Claims A2 and A3 (Appendix A), changing prices $(p, q)$ by $\left(p^{T}, q^{T}\right)$, and taking the limit as $T$ goes to infinity.

Thus, since $\sum_{\xi \in D \backslash D^{t-1}} v_{\xi}^{h}\left(\bar{z}_{\xi}^{h}\right) \leq \sum_{\xi \in D \backslash D^{t-1}} u_{\xi}^{h}\left(\mathbb{W}_{\xi}\right)$, we have $\lim _{t \rightarrow+\infty} \sum_{\xi \in D_{t}} \bar{\gamma}_{\xi}^{h} \nabla_{2} g_{\xi}^{h}(\bar{p}, \bar{q}) \bar{z}_{\xi^{-}}^{h}=0$. Moreover, $\left(\bar{\gamma}_{\xi}^{h} \nabla_{1} g_{\xi}^{h}(\bar{p}, \bar{q})+\sum_{\mu \in \xi^{+}} \bar{\gamma}_{\mu}^{h} \nabla_{2} g_{\mu}^{h}(\bar{p}, \bar{q})\right) \in \partial^{+} v^{h}\left(\bar{z}_{\xi}^{h}\right)$. By the same arguments made in the proof of Proposition 1-(i) (see Appendix A) we conclude that Euler equations and transversality conditions hold. Therefore, it follows from Proposition 1-(ii) that the allocation $\left(\bar{z}_{\xi}^{h}\right)_{\xi \in D}$ is optimal for agent $h \in H$, which concludes the proof of the Theorem 1.

\section{REFERENCES}

[1] Araujo, A., J. Fajardo, and M.R. Páscoa (2005): "Endogenous Collateral ," Journal of Mathematical Economics, 41, 439-462.

[2] Araujo, A., M.R. Páscoa, and J.P. Torres-Martínez (2005): "Bubbles, Collateral and Monetary Equilibrium," working paper No 513, PUC-Rio.

[3] Araujo, A., M.R. Páscoa, and J.P. Torres-Martínez (2002): "Collateral Avoids Ponzi Schemes in Incomplete Markets," Econometrica 70, 1613-1638.

[4] Bewley, T. (1980): "The Optimum Quantiy of Money," in Models of Monetary Economics, ed. by J. Kareken and N. Wallace. Minneapolis: Federal Reserve Bank.

[5] Friedman, M. (1969): "The Optimum Quantity of Money," Aldine Publishing Company, Chicago.

[6] Geanakoplos, J. and W.R. Zame (2002): "Collateral and the Enforcement of Intertemporal Contracts," Yale University Working Paper.

[7] Gale, D. (1973): "Pure Exchange Equilibrium of Dynamic Economic Models," Journal of Economic Theory, 6, $12-36$.

[8] Gimenez, E. (2007): "On the Positive Fundamental Value of Money with Short-Sale Constraints: A Comment on Two Examples," Annals of Finance, 3, 455-469.

[9] Gimenez, E. (2003): "Complete and incomplete markets with shot-sale constraints," Economic Theory, 21, 195204.

[10] Grandmont, J.-M., and Y. Younès (1972): "On the Role of Money and the Existence of a Monetary Equilibrium," Review of Economic Studies, 39, 355-372.

[11] Grandmont, J.-M., and Y. Younès (1973): "On the Efficiency of a Monetary Equilibrium," Review of Economic Studies, 40, 149-165.

[12] Hernandez, A., and M. Santos (1996): "Competitive Equilibria for Infinite-Horizon Economies with Incomplete Markets," Journal of Economic Theory, 71, 102-130.

[13] Kubler, F. and K. Schmedders (2003): "Stationary equilibria in asset pricing models with incomplete markets," Econometrica, 71, 1767-1793.

[14] Magill, M., and M. Quinzii (1996): "Incomplete Markets over an Infinite Horizon: Long-lived Securities and Speculative Bubbles," Journal of Mathematical Economics, 26, 133-170.

[15] Rockafellar, R.T. (1997): “Convex Analysis," Princeton University Press, Princeton, New Yersey.

[16] Samuelson, P. (1958): "An Exact Consumption-Loan Model of Interest with or without the Social Contrivance of Money," Journal of Poltical Economy, 66, 467-482.

[17] Santos, M. (2006): "The Value of Money in a Dynamic Equilibrium Model" Economic Theory, 27, 39-58.

[18] Santos, M., and M. Woodford (1997): "Rational Asset Pricing Bubbles," Econometrica, 65, 19-57.

[19] Santos, M., and M. Woodford (1996): "A Value Theory of Money," mimeograph, ITAM, México, DF. 
[20] Santos, M., and M. Woodford (1992): “A Value Theory of Money," mimeograph, Universidad Carlos III de Madrid.

[21] Woodford M. (1994): "Monetary Policy and Price Level Determinacy in a Cash-in-Advance Economy," Economic Theory, 4, 345-380.

IMPA AND EPGE/FGV

Estrada Dona Castorina 110, 22460-320, Rio de Janeiro, Brazil.

E-mail address: aloisio@ impa.br

Faculdade de Economia, Universidade Nova de Lisboa

Travessa Estevão Pinto, 1099-032, Lisbon, Portugal.

E-mail address: pascoa $@$ fe.unl.pt

Department of Economics, University of Chile

Diagonal Paraguay 257 office 1604, Santiago, Chile.

E-mail address: jptorres.martinez $@$ gmail.com 